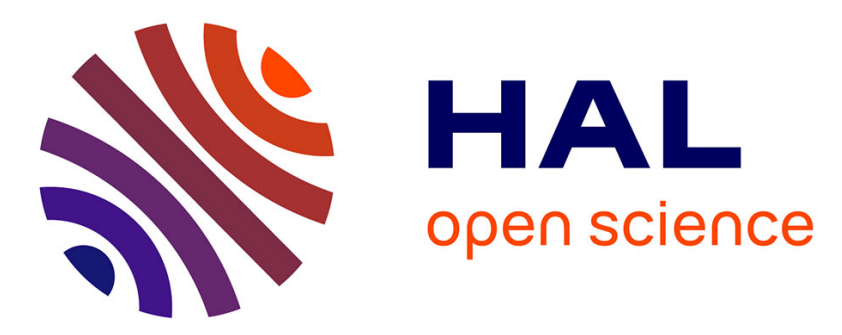

\title{
A penalty model of synthetic micro-jet actuator with application to the control of wake flows \\ Richard Pasquetti, Noele Peres
}

\section{To cite this version:}

Richard Pasquetti, Noele Peres. A penalty model of synthetic micro-jet actuator with application to the control of wake flows. Computers and Fluids, 2015, pp.203-217. 10.1016/j.compfluid.2015.02.019 . hal-01144703

\section{HAL Id: hal-01144703 \\ https://hal.univ-cotedazur.fr/hal-01144703}

Submitted on 20 Oct 2016

HAL is a multi-disciplinary open access archive for the deposit and dissemination of scientific research documents, whether they are published or not. The documents may come from teaching and research institutions in France or abroad, or from public or private research centers.
L'archive ouverte pluridisciplinaire HAL, est destinée au dépôt et à la diffusion de documents scientifiques de niveau recherche, publiés ou non, émanant des établissements d'enseignement et de recherche français ou étrangers, des laboratoires publics ou privés. 


\title{
A penalty model of synthetic micro-jet actuator with application to the control of wake flows
}

\author{
Richard Pasquetti ${ }^{1}$ and Noele Peres \\ Laboratoire J. A. Dieudonné Université de Nice-Sophia Antipolis \\ Parc Valrose, F-06108 Nice Cedex 02, France.
}

\begin{abstract}
A simple model of synthetic (zero-net mass-flux) micro-jet is proposed and implemented in a large-eddy simulation spectral solver of the incompressible Navier-Stokes equations. Essentially, it is simply required to introduce a penalty like term in the momentum equation, with a variable penalty coefficient that culminates during the expulsion phase. Examples of applications are considered for the control of several turbulent wake flows, the backward facing step, the D-shaped body and the square back Ahmed body.
\end{abstract}

Keywords:

Flow control, jet actuators, synthetic jets, turbulent wakes, large-eddy simulations, penalty techniques, spectral vanishing viscosity

\section{Introduction}

An attractive perspective to control wake flows is based on the use of micro-jets. This is especially of interest in the frame of the automotive industry, in view of reducing the drag coefficient and so the consumption of fossil energy. An efficient strategy has however not yet emerged and the questions remain numerous, e.g. related to the implantation of the micro-jets or to the adhoc frequency in case of synthetic micro-jets. This is due to the fact that the physics involved is far from obvious, that experiments are not easy to carry out and that the numerical simulations remain extremely challenging, see e.g. the review papers $[1,2]$. Here we are interested in the numerical approach.

Detailed simulations on the flow generated by one micro-jet into an open medium have already been investigated, see e.g. $[3,4,5,6]$. Here we are rather interested in studying the influence of micro-jets on complex flows, i.e. three-dimensional, unsteady and possibly turbulent, like it occurs for the wake generated by cars. In this case a multiscale problem arises, and it is then required to develop simplified models of

\footnotetext{
${ }^{1}$ Corresponding author, tel.:+33(0)4 920762 08, fax:+33(0)4 935179 74, email: richard.pasquetti@unice.fr
} 
the micro-jets, easy to implement in existing codes together with providing reliable results. Such a research is certainly not new, but up to our knowledge the modelling issue is far from closed, see e.g. the report [7] which points out large variations among the CFD (Computational Fluid Dynamics) results or [8] where the actuator geometry is considered but with simplified boundary conditions. The present work follows some existing approaches, see e.g. $[9,10,11,12]$. A surface boundary condition is proposed in [10]. In [11] the actuators are modelled by introducing virtual forces that bring the fluid to a specified velocity. In [12], where compressible flows are considered, mass, momentum and energy sources are introduced in the governing equations to model the actuators. In [9], a penalty technique is used to enforce the micro-jet velocity in a given finite volume of the mesh.

Rather than using a micro-jet model one may think to use a mesh refinement strategy, see e.g. [13, 14]. Then, although refined, the mesh remains generally rough with respect the micro-jet geometry. The question is then to know which approach is preferable between the modelling and the crude mesh refinement ones. The answer is far from obvious and certainly problem dependent. When many micro-jets are involved, the mesh refinement approach generally becomes unrealistic. Moreover, the computational cost is then extremely high, e.g. due to the coupling between the mesh size and the time step through a CFL (Courant-Friedrichs-Lewy) condition.

Our approach is based on a modelling of the micro-jets by an adhoc source term of momentum. In Section 2 we develop and justify this modelling. In Section 3 we explain how such a model can be implemented in an existing spectral LES (large-eddy simulation) solver of the incompressible Navier-Stokes equations (SVVLES code). Results are presented for the 3D (three-dimensional) backward facing step turbulent flow, for the 3D D-shaped body and for the 3D square back Ahmed body wake flows in Section 4. This work takes indeed part of efforts recently devoted to the drag reduction of the Ahmed body by active flow control, see $[9,15,16,17,18]$. We conclude in Section 5.

\section{Micro-jet modelling}

We are interested by the influence of micro-jets on the three-dimensional turbulent wakes generated by a bluff body. Because the scale of the micro-jet is generally of the order of the $m m$, whereas the one of the bluff body is typically of the $m$, a modelling of the micro-jet is required. In two previous papers [19, 20], we proposed to model the micro-jets by enforcing the velocity at some grid-points located in the immediate vicinity of the micro-jet aperture. This was done using a penalization technique. Such an approach shows however the two following drawbacks: (i) The micro-jet is only characterized by the expulsion velocity, so 
that its flow rate (or intensity) is not taken into account; (ii) The stiff velocity gradient which develops at the micro-jet aperture is not considered. Here we propose a more relevant micro-jet modelling, based on punctual sources of mass and momentum, and we associate the latter to the hydrodynamic impulse force of the synthetic micro-jet during the expulsion phase.

The mass and momentum conservation equations with source terms, $f$ and $\boldsymbol{m}$ respectively, can be written as:

$$
\begin{aligned}
& \partial_{t} \rho+\nabla \cdot(\rho \boldsymbol{u})=f \\
& \partial_{t}(\rho \boldsymbol{u})+\nabla \cdot(\rho \boldsymbol{u} \boldsymbol{u}+p \mathbb{I}-\sigma)=\boldsymbol{m}
\end{aligned}
$$

with $\mathbb{I}$ for the identity tensor, $t$ for the time and where $\rho, \boldsymbol{u}, p$ and $\sigma$ denote the density, velocity field, pressure field and viscous tensor, respectively. For incompressible Newtonian fluids, this yields:

$$
\begin{aligned}
& \nabla \cdot \boldsymbol{u}=f / \rho \\
& \partial_{t} \boldsymbol{u}+\boldsymbol{u} \cdot \nabla \boldsymbol{u}=-\nabla(p / \rho)+\nu \Delta \boldsymbol{u}+(\boldsymbol{m}-f \boldsymbol{u}) / \rho
\end{aligned}
$$

where $\nu$ is the kinematic viscosity.

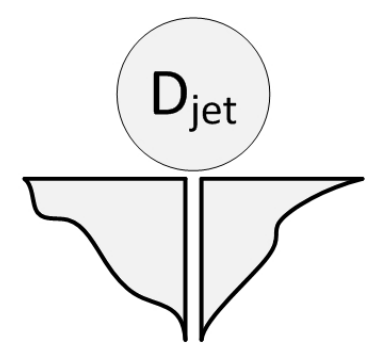

Figure 1: Micro-jet modelling: Visualization of the volume $D_{j e t}$ associated to the micro-jet.

We have now to define precisely the mass and momentum source term, $f$ and $\boldsymbol{m}$, to be used to model the presence of the micro-jets. To this end we consider a single micro-jet and the control volume $D_{j e t}$ located at its aperture, see Fig. 1. Later on, this control volume will correspond to a computational cell. Because we do not want to adjust the computational mesh to the micro-jet geometry, we define $f_{j e t}$ as the mass source term supported by $D_{j e t}$ that takes into account the mass injected into the fluid by the micro-jet:

$$
\int_{D_{j e t}} f_{j e t} d \Omega=q_{m}
$$


where $q_{m}$ is the mass flow rate.

From Euler theorem, the impact force of the jet onto the fluid essentially equals $\boldsymbol{F}=q_{m} \boldsymbol{u}_{j e t}$, where $\boldsymbol{u}_{j e t}$ is the velocity inside the micro-jet (inertial terms are neglected). We suggest to transform this momentum flux term into a volume source term, such that:

$$
\int_{D_{j e t}} \boldsymbol{m}_{j e t} d \Omega=\boldsymbol{F}
$$

Since we do not plan to use a very fine grid at the micro-jet aperture, in practice $D_{j e t}$ will only contain one grid-point and so corresponds to the local computational cell. In this case, in the spirit of finite volume approaches, one may set:

$$
\begin{aligned}
& \int_{D_{h}} f_{\text {jet }} d \Omega \approx\left|D_{h}\right| f_{j e t}(P)=q_{m} \\
& \int_{D_{h}} \boldsymbol{m}_{\text {jet }} d \Omega \approx\left|D_{h}\right| \boldsymbol{m}_{\text {jet }}(P)=\boldsymbol{F}
\end{aligned}
$$

where $D_{h} \equiv D_{\text {jet }}$ corresponds to the local cell, $\left|D_{h}\right|$ to its volume and $P$ to its center. In this case:

$$
\begin{aligned}
& f_{\text {jet }}(P)=\rho \frac{q_{v}}{\left|D_{h}\right|} \\
& \boldsymbol{m}_{\text {jet }}(P)=\rho \frac{q_{v}}{\left|D_{h}\right|} \boldsymbol{u}_{\text {jet }}=f_{\text {jet }}(P) \boldsymbol{u}_{\text {jet }}
\end{aligned}
$$

where $q_{v}$ is the volume flow rate of the micro-jet $\left(q_{v} /\left|D_{h}\right|\right.$ has the dimension of an inverse of time).

In any cases $D_{j e t}$ should be associated to the mesh in order to carry out easily the quadrature involved in eq. (8). Using $D_{j e t} \not \equiv D_{h}$ may be of interest, e.g. to regularize the numerical problem. In view of regularization one has however to choose efficient ansatz for $f_{j e t}(\boldsymbol{x}, t)$ and $\boldsymbol{m}_{\text {jet }}(\boldsymbol{x}, t)$ in $D_{\text {jet }}$. This is strongly linked to the numerical method at hand. In the present paper we restrict ourselves to the case where $D_{j e t} \equiv D_{h}$ is only associated to one computational cell.

Let us now consider the case of synthetic micro-jets. In this case $f_{j e t}$ shows a periodic behavior. However, it is clear that the expulsion phase strongly differs from the ingestion one, e.g. as pointed out by the simulation results obtained by [3] for a single micro-jet acting on a quiet fluid. In the expulsion phase one clearly observes a jet like flow, whereas in the ingestion phase the fluid remains essentially quiet, except in the immediate vicinity of the micro-jet aperture. This is associated to the fact that the micro-jet mechanism is active in the expulsion phase and on the contrary passive in the ingestion one, which may be typical of synthetic 
micro-jets.

To cope with this behavior, we suggest to apply the momentum forcing in the expulsion phase and to assume that in the ingestion phase the momentum forcing just compensates the inertial term. Defining the flow rate as positive in the expulsion phase, from (9)-(10) this resumes to consider in eq. (4) the following momentum source term:

$$
\left(\boldsymbol{m}_{\text {jet }}(P)-f_{j e t}(P) \boldsymbol{u}\right) / \rho=\frac{q_{v}^{+}}{\left|D_{h}\right|}\left(\boldsymbol{u}_{j e t}-\boldsymbol{u}\right)
$$

where $q_{v}^{+}=\max \left(q_{v}, 0\right)$.

Let us remark that in the $1 \mathrm{D}$ approximation, $q_{v}= \pm\left|\boldsymbol{u}_{j e t}\right| s_{j e t}$, where $s_{j e t}$ is the surface section of the micro-jet. As expected, $\left|\boldsymbol{m}_{j e t}\right|$ is thus proportional to $\boldsymbol{u}_{j e t}^{2}$. Since $\partial_{t} \boldsymbol{u}_{j e t}^{2}=2 \boldsymbol{u}_{j e t} \cdot \partial_{t} \boldsymbol{u}_{j e t}$, in case of synthetic micro-jets one observes that $q_{v}^{+}\left|\boldsymbol{m}_{\text {jet }}\right|$ shows a smooth $C^{1}$ time variation. Thus, if $\left|\boldsymbol{u}_{\text {jet }}\right|$ is proportional to $\sin (\omega t)$, where $\omega$ is the micro-jet angular frequency, then $q_{v}^{+}\left|\boldsymbol{m}_{j e t}\right|$ is proportional to $\sin ^{2}(\omega t)$ in the expulsion phase.

Taking into account the source term $f_{\text {jet }}$ in the continuity equation is in practice difficult, especially when high order methods are concerned. Moreover, the synthetic jets are by definition zero mass flux net over a period and the mass fluctuations, due to the expulsion-ingestion process, are generally weak since micro-jets are concerned. This is why we suggest (i) to simply relax the divergence free constraint at the grid-point where the jet is considered and (ii) to apply the forcing at two grid-points away from the physical boundary. Then, one can look at eq. (11) as a penalty term, i.e. that enforces $\boldsymbol{u}=\boldsymbol{u}_{j e t}$ if $q_{v} \gg 1$ or if $\left|D_{h}\right| \ll 1$, with a penalization coefficient $q_{v}^{+} /\left|D_{h}\right|$ that depends on the micro-jet instantaneous flow rate.

Details on the practical implementation of this penalty model are provided in next Section.

\section{Implementation in an existing LES solver}

Here we explain how the proposed modelling of the micro-jets has been implemented in an existing LES solver. The SVVLES spectral solver allows the computation of incompressible turbulent wake flows. Details may be found in [21]. To summarize, it makes use:

- of a multi-domain spectral approximation in space. To consider domains of large aspect ratio, a domain decomposition technique is implemented in the streamwise direction. In each subdomain, a collocation Chebyshev - Fourier Galerkin approximation is used (the transverse direction is assumed periodic).

- of a three-step time-stepping. First, the advection terms are considered explicitly using an Operator 
Integration Factor (OIF) semi-Lagrangian method and a forth order Runge-Kutta (RK4) algorithm. Second, the diffusion terms are treated implicitly, using a Second order Backward Difference Formula (BDF2) approximation of the time derivative. Third, an incremental projection method is used to get a divergence free velocity field.

- The LES capability is implemented in the diffusion step, by supplementing the momentum equation with an additional Spectral Vanishing Viscosity (SVV) term only active on the high frequency range of the velocity field.

- The modelling of the obstacle is based on a volume penalization technique, namely the pseudopenalization technique described in [22]. In this approach the penalization term is not explicitly expressed but introduced via the time-scheme, so that inside the obstacle the flow is governed by the steady Stokes equations penalized with a $C=\alpha / \tau$ coefficient, $\tau$ being the time-step and with $\alpha=3 / 2$ for the BDF2 scheme, see Appendix A.

To check the capabilities of the approach described in Section 2, we first consider the case of a punctual forcing in a 2D fluid at rest. For the momentum source term we use the formulation (11), with $D_{h}=h_{x} h_{y}$ and where $h_{x}$ and $h_{y}$ are the local space steps. As already discussed, such an approach is of course better justified in the frame of a finite volume method, since source terms are then assumed constant in the computational cell. In the frame of a spectral method, one may think to restart from (8) and use a more sophisticated quadrature rule, i.e. exact for the polynomial interpolant of $\boldsymbol{m}(P)$, but this polynomial interpolant is not compactly supported by $D_{h}$. This is why we prefer to retain the proposed quadrature approximation.

As mentioned above, the divergence free constraint is enforced by projection, i.e. at time $t_{n}$ we first compute a non solenoidal velocity field $\boldsymbol{u}^{\star}$ and then use the Helmhotz decomposition $\boldsymbol{u}^{\star}=\boldsymbol{u}^{n}+\tau \nabla \varphi$, to compute the pressure update $\varphi$ which solves:

$$
\tau \Delta \varphi=\nabla \cdot \boldsymbol{u}^{\star}-\nabla \cdot \boldsymbol{u}^{n}
$$

In the frame of a collocation method $\nabla \cdot \boldsymbol{u}^{n}$ must equal 0 at all grid-points except those associated to a punctual mass source. For the reasons explained in Section 2 we do not take into account the mass source in the equation for $\varphi$ and simply relax the divergence free constraint. This is done by not enforcing the correction $\boldsymbol{u}^{n}=\boldsymbol{u}^{\star}-\tau \nabla \varphi$ at the grid-points where a forcing is introduced.

To visualize the effect of the single jet in a fluid at rest, Fig. 2 provides the vertical component of the 


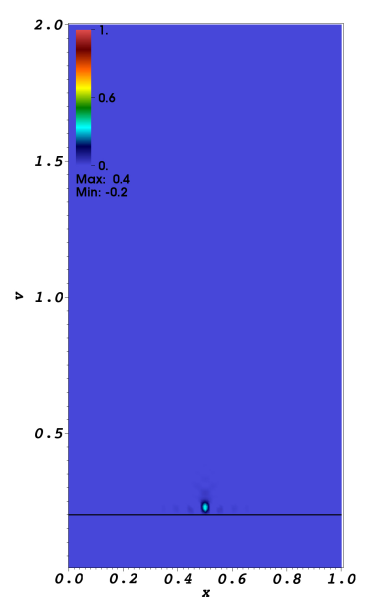

(a) $t=1$

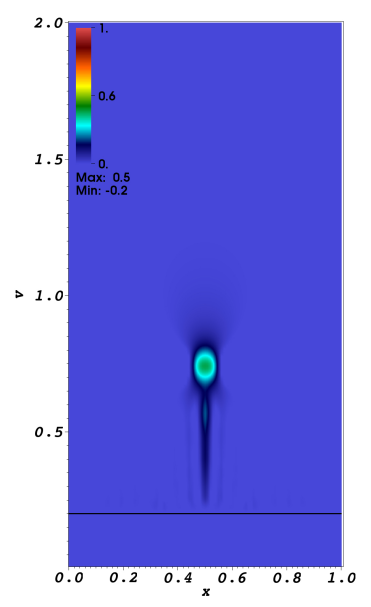

(e) $t=5$

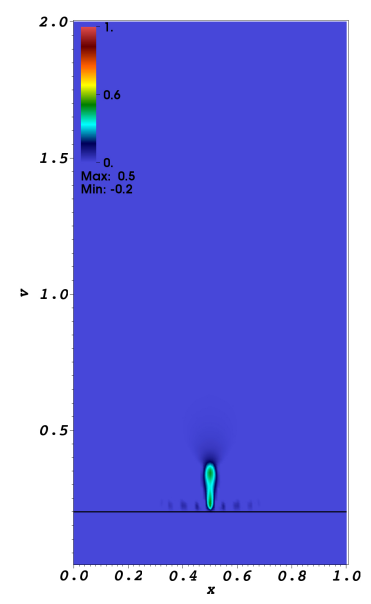

(b) $t=2$

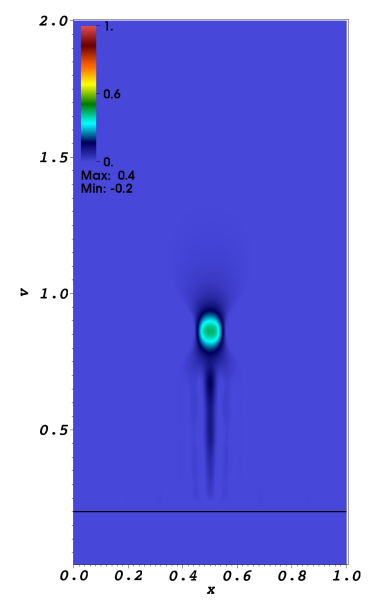

(f) $\mathrm{t}=6$

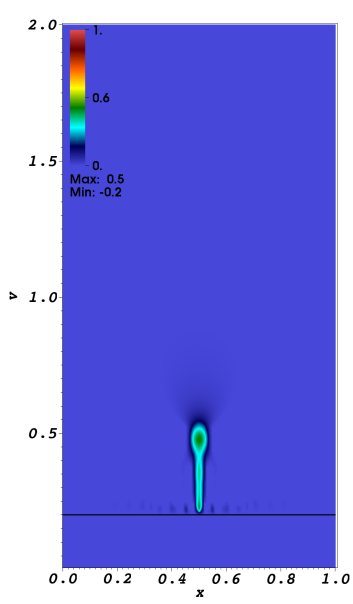

(c) $t=3$

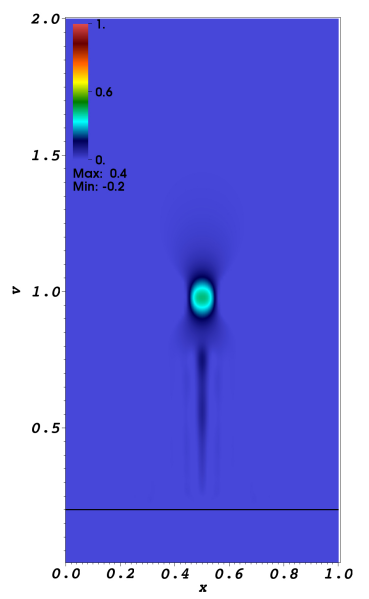

(g) $\mathrm{t}=7$

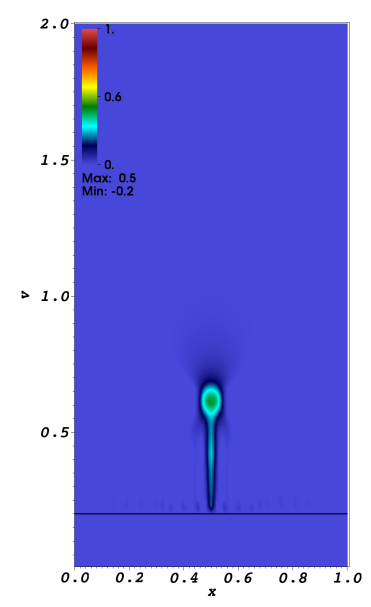

(d) $\mathrm{t}=4$

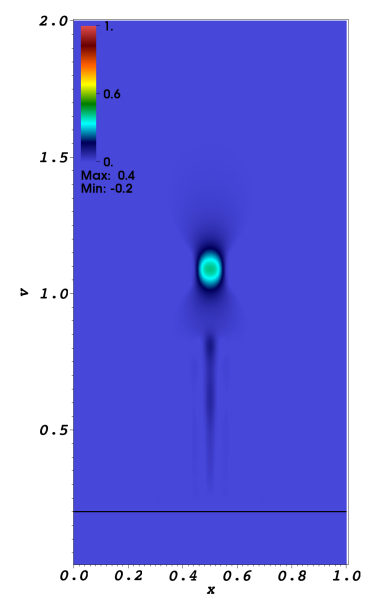

(h) $t=8$

Figure 2: Snapshots of the vertical velocity component $v \equiv u_{y}$ at different times.

velocity at different times. At the aperture of the actuator, $q_{j e t}=s_{j e t} \sin \left(2 \pi S t_{j e t} t\right)\left(q_{j e t}\right.$, dimensionless volume flow rate) and $\boldsymbol{u}_{j e t}=\sin \left(2 \pi S t_{j e t} t\right) \boldsymbol{e}_{y}$, with $s_{j e t}=10^{-2}$ for the micro-jet section and $S t_{j e t}=0.1082$ for the micro-jet Strouhal number. The wall is modelled using the pseudo-penalization technique. The forcing is punctual and applied at two grid-points above the wall, i.e. along the line $x=0.5$ there is one free grid-point between the last grid-point involved in the wall modelling and the grid-point where the punctual forcing acts. As expected, one observes the vertical motion of the fluid perturbation.

More quantitatively, one can look at the evolution of the vertical velocity at a point above the actuator. In Fig. 3 we present such a result for the point $(x=0, y=0.305)$ and moreover consider four different configurations: 


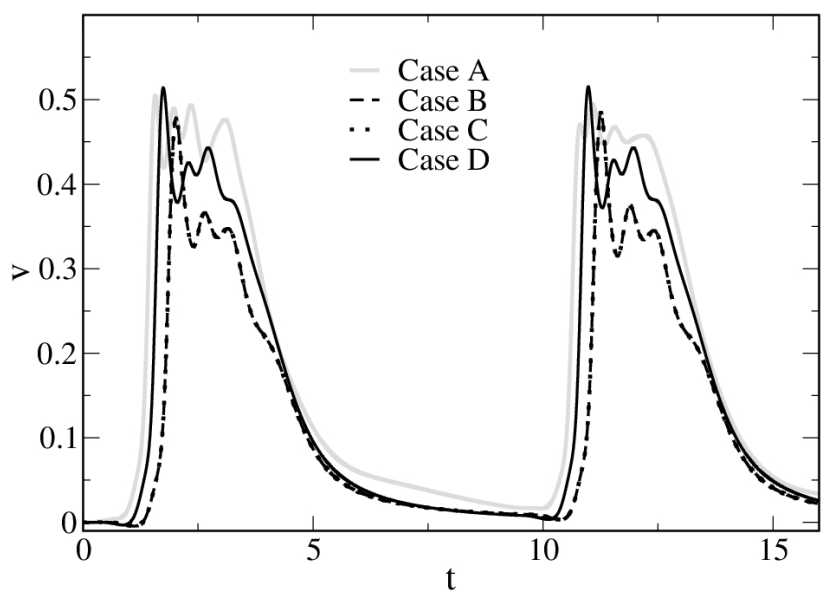

Figure 3: Time evolution of the vertical velocity component $v \equiv u_{y}$ at the points $(x=0.5, y \approx 0.3)$. A: Without wall, B: the punctual forcing is applied at one grid-point from the wall. C: idem B, but without relaxation of the divergence free constraint. $\mathrm{D}$ : idem B, but the punctual forcing is applied at two grid-points from the wall.

A : There is no wall and the forcing term (11) is thus applied at an isolated grid-point.

B : The forcing is applied at one grid-point away from the horizontal wall which is modelled using the pseudo-penalization technique; Since there is no free grid-point between the wall and the point where the forcing is applied, we use $\left|D_{h}\right|=h_{x} h_{y} / 2$.

C : This configuration is similar to the configuration (B), but without using the proposed relaxation of the divergence free constraint.

D : As suggested in Section 2 the forcing is applied at two grid-points away from the horizontal wall and $\left|D_{h}\right|=h_{x} h_{y}$.

The graph of Fig. 3 points out that the presence of one free grid-point is important in the present modelling. On the contrary, the fact that for the cases $\mathrm{B}$ and $\mathrm{C}$ the curves coincide, rather shows that relaxing the divergence free constraint at the grid-point where the forcing is applied is of minor importance and so mainly satisfactory from the theoretical point.

Since the penalty term defined by (11) depends on the space discretization, through $\left|D_{h}\right|$, it is of interest to compare the results obtained for different discretizations. Fig. 4 compares the evolution of the vertical velocity at the point $(x=0.5, y \approx 0.3)$ (the exact $y$-value is mesh dependent) for three different discretizations, $N=(40,254)$, i.e. the discretization used previously, $N=(28,178)$ and $N=(56,358)$, i.e. associated to about twice less and about twice more grid-points, respectively. Clearly, due to the penalty feature of the micro-jet modelling term, the results are only slightly dependent on the space discretization. 


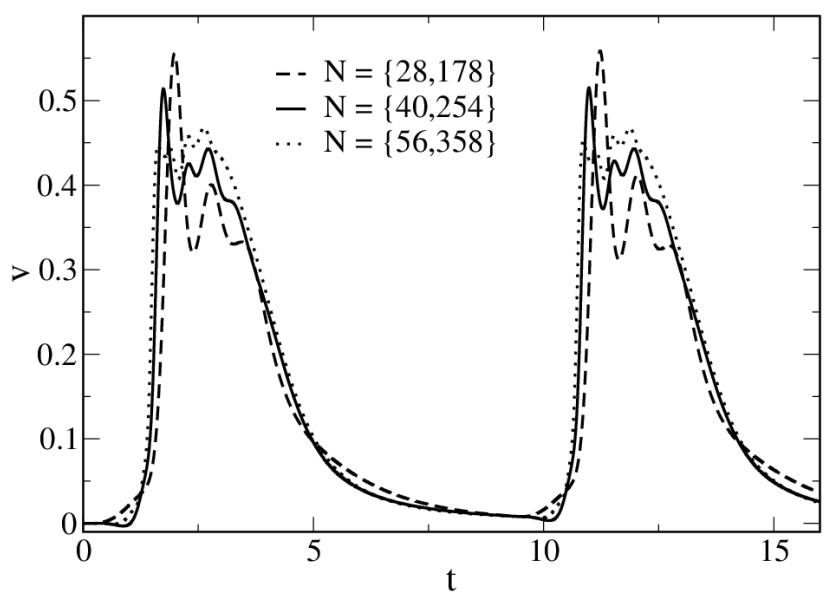

Figure 4: Time evolution of the vertical velocity component $v \equiv u_{y}$ at $(x=0.5, y \approx 0.3)$ for three different spatial discretizations.

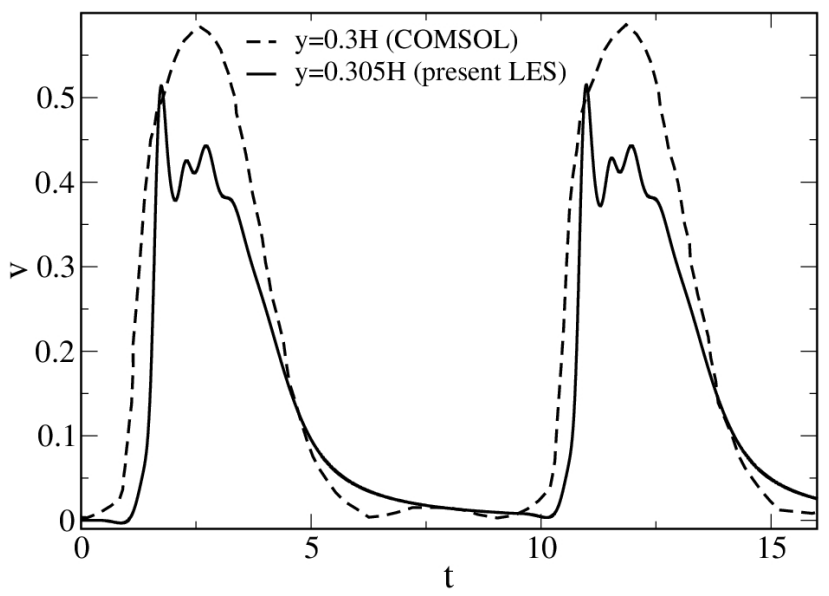

Figure 5: Time evolution of the vertical velocity component $v \equiv u_{y}$ at $(x=0.5, y=0.3)$ using the present modelling and a detailed description of the actuator.

For the considered problem, i.e. the injection of fluid in a 2D flow at rest, a detailed computation can be carried out. Fig. 5 compares the vertical velocity at $y=0.3$ using the proposed modelling with the one obtained using the COMSOL software, when taking into account precisely the injector [3]. The discretization is here again $N=(40,254)$. When taking in mind that in our approach the micro-jet is simply modelled by a punctual force term, the agreement may be considered satisfactory. A better agreement may still been obtained by adjusting the volume $\left|D_{h}\right|$ associated to the source of momentum, or equivalently by introducing a "calibration constant", see e.g. [12], but we think preferable to preserve the parameter free feature of our modelling. 


\section{Applications}

Here we apply the previous modelling of synthetic micro-jets to three different test-cases. The first one considers the turbulent backward facing step flow, with the objective to reduce the recirculation length. The two others are concerned with the reduction of the drag coefficient of motor vehicles. They focus on the D-shaped body and on the Ahmed body with vertical slant.

\subsection{Backward facing step}

We consider a classical test-case whose topic is the control of the backward facing step flow using a synthetic micro-jet. The actuator is located close to the end side of the step with an inclination angle of $45^{\circ}$, see e.g. $[23,24,25,26]$ for the experiments and $[27,28,29,30,31]$ for the numerical approaches. In [23] the experiments are carried out for $13000 \leq R e \leq 33000$ whereas in $[24,25,26]$ the Reynolds number equals $R e=3700$. In these studies it is pointed out that the micro-jet induces an increase of the level of turbulence in the shear layer bordering the recirculation bubble and thus a shortening of the recirculation length. More details on the physical mechanism may be found in both the experimental and numerical papers. Concerning the numerical approach, in [31] it is checked that LES approaches are required to capture a shortening of the recirculation up to $30 \%$, i.e. comparable to the one obtained in the experiments.

Here the Reynolds number, based on the height $h$ of the step and the inlet velocity maximum, equals $R e=33000$. Downstream of the step the channel is of dimensionless height 3 and in the periodic spanwise direction it is assumed of width 2. Such a choice of channel width may be justified by the LES results, at $R e=3700$, presented in [27] (see Fig. 2), where the auto-correlation coefficient in spanwise direction of the streamwise velocity approximately vanishes at $0.25 \mathrm{~h}$. The length of the channel is taken large enough to be sure that at the outlet the flow is outgoing. At the lower boundary, i.e. at the wall, we use a no-slip condition, whereas at the upper boundary we use a free-slip condition. At the outlet, we use an advection condition based on the mean flow velocity as Outflow Boundary Condition (OBC). We have observed that the results were sensitive to the inlet velocity profile. In the spirit of [28], where the same geometry and Reynolds number are considered, here it is steady and described by (in dimensionless form):

$$
U_{\text {in }}\left(y^{*}\right)=1.967\left[\frac{y^{*}}{10}\right]^{0.25}, \text { for } 0 \leq \mathrm{y}^{*} \leq 2 / 3
$$

where $y^{*}=(y-1) / 3$.

The step, of dimensionless length 1.53 is modelled by using the pseudo-penalization technique [22], see Appendix A. The micro-jet is modelled as previously described, with $\boldsymbol{u}_{\text {jet }}=U_{\text {jet }}(\cos (\pi / 4), \sin (\pi / 4), 0) \sin \left(2 \pi S t_{\text {jet }} t\right)$, 
with $U_{j e t}$ for the amplitude and $S t_{j e t}$ for the dimensionless frequency. The thickness of the micro-jet is taken equals to $s_{j e t}=0.02$ and we assume, with $u_{j e t}= \pm\left|\boldsymbol{u}_{j e t}\right|$ (with the convention $u_{j e t}$ positive in the expulsion phase), $q(t)=u_{\text {jet }} s_{j e t}$ per spanwise unit length.
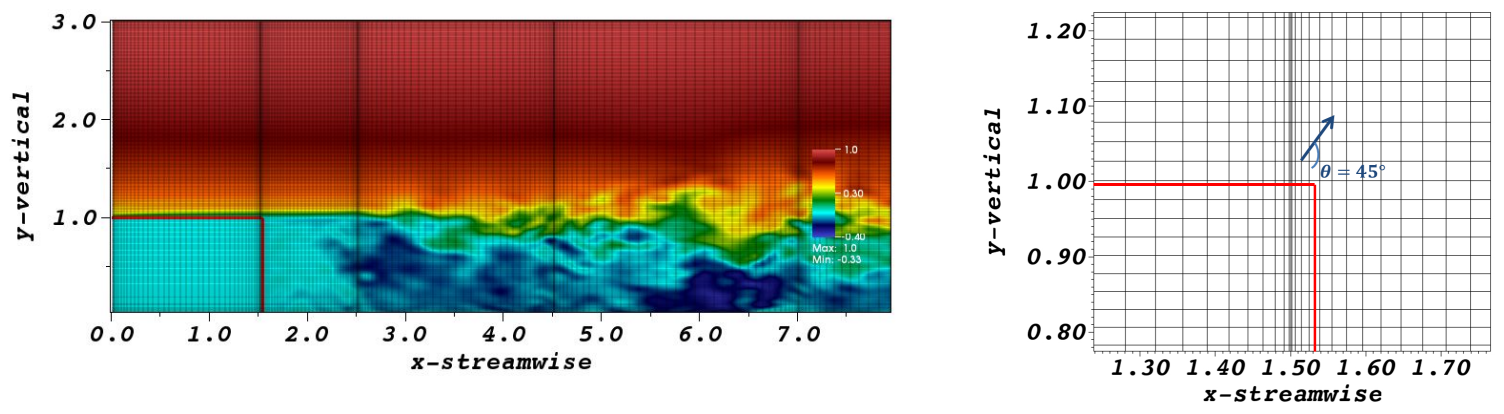

Figure 6: Instantaneous streamwise velocity together with the mesh in the upstream part of the computational domain (left). Zoom of the mesh at the edge of the step (right).

Preliminary computations have been carried out in the $2 \mathrm{D}$ channel like geometry $\Omega=(0,24) \times(0,3)$. Despite the fact that these computations were carried out during a large interval on time of 4000 dimensionless time units, in order to get converged statistics, the results were not satisfactory: The decrease of the recirculation length was smaller than in the experiments of [23], and very irregular with respect to the expulsion frequency. Although 2D LES and 2D RANS (Reynolds-Averaged Navier-Stokes) approaches were used in $[28,30]$, respectively, we have guessed that $3 \mathrm{D}$ computations were required to better fit with the experiments. Note that 2D RANS approaches are justified by the fact that the mean quantities are indeed 2D, but a priori this justification fails when filtered fluctuating quantities are computed.
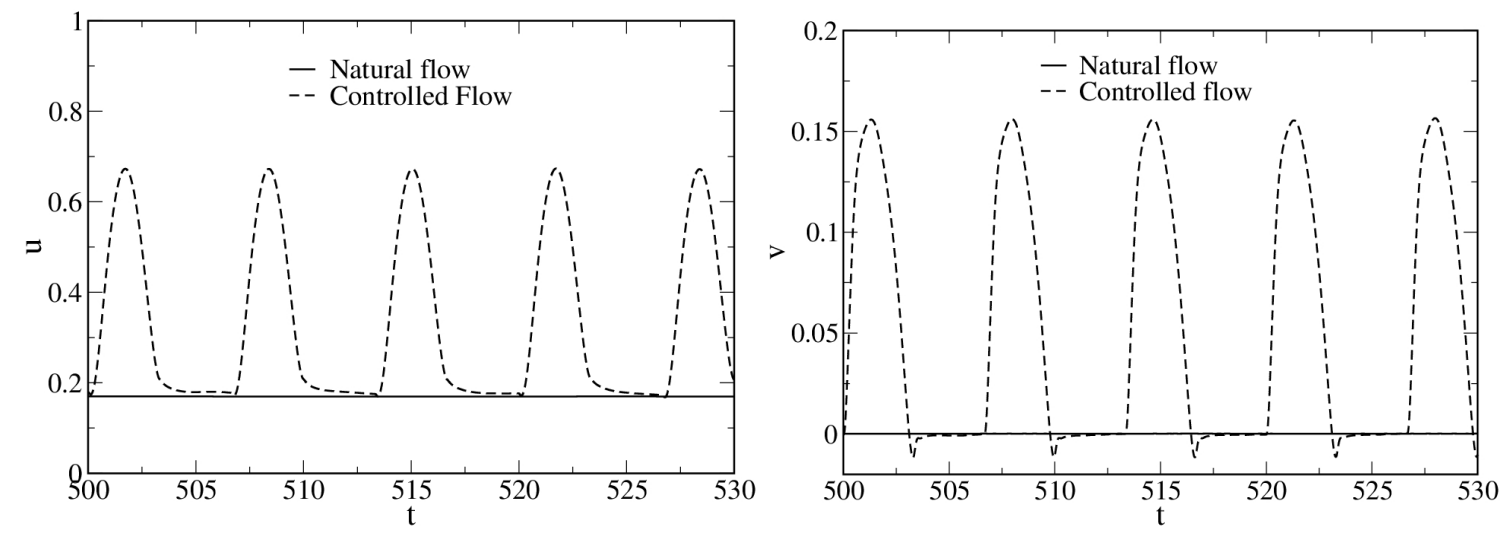

Figure 7: Time variations of the velocity at the grid-point where the forcing term (11) applies. Streamwise component $u \equiv u_{x}$ (left) and vertical component $v \equiv u_{y}$ (right).

3D computations have been carried out in the channel like geometry $\Omega=(0,43) \times(0,3) \times(0,2)$, using 16 
subdomains and a polynomial approximation degree $N=\{40,120,32\}$ in each of them, resulting in about 4.96 millions of grid-points (note that 64 grid-points are associated to the trigonometric polynomial degree 32). An instantaneous snapshot of the streamwise velocity together with the mesh is shown in Fig. 6(left). In the zoom provided in Fig. 6(right), the micro-jet modelling is visualized: The punctual forcing (11) is located close to the end side of the step and at two grid-points away from the horizontal wall. The domain $D_{h}=h_{x} h_{y}$, per spanwise unit length, makes use of the local values of $h_{x}$ and $h_{y}$. Note that the step was located just downstream of a subdomain interface in order to avoid any numerical artifacts associated to a possible interaction with the micro-jet flow. Fig. 7 shows, for the dimensionless micro-jet frequency $S t_{j e t}=0.15$ (micro-jet Strouhal number), the evolutions of the $x$ and $y$ components of the velocity at the grid-point where the forcing (11) applies.
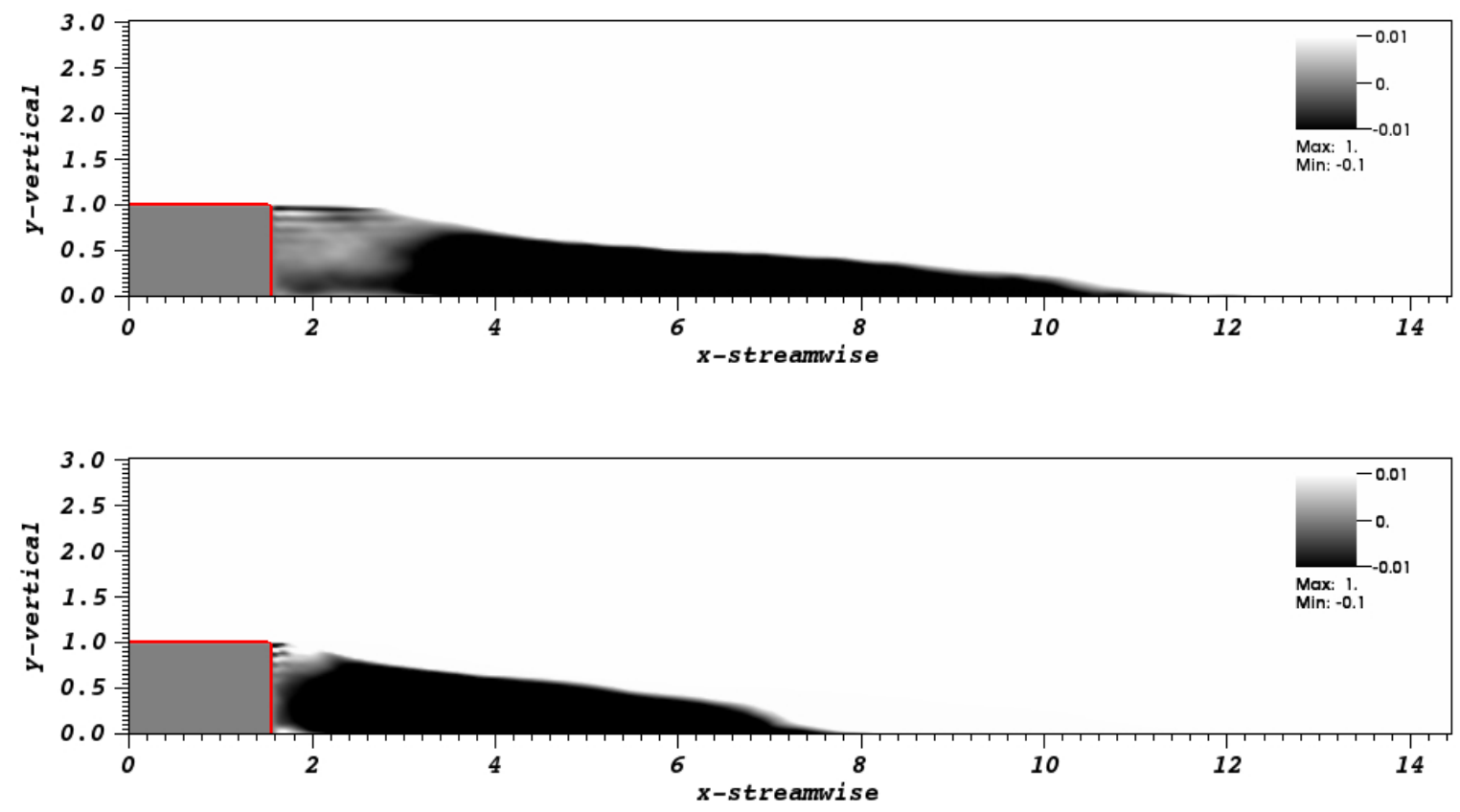

Figure 8: Mean streamwise velocity for the natural flow (top) and for the controlled flow, with $U_{\text {jet }}=1.5$ and $S t_{j e t}=0.15$ (bottom).

Fig. 8 compares the mean streamwise component of the flow, as obtained for the natural flow and for the controlled flow with $S t_{j e t}=0.15$. The mean quantities have been obtained using a time-averaging over 400 time units completed with an additional space averaging along the homogeneous $z$-direction. To better appreciate the recirculation length the negative and positive values are saturated (range taken between $\pm 0.01)$. As expected, one clearly observes the decrease of the recirculation length. 


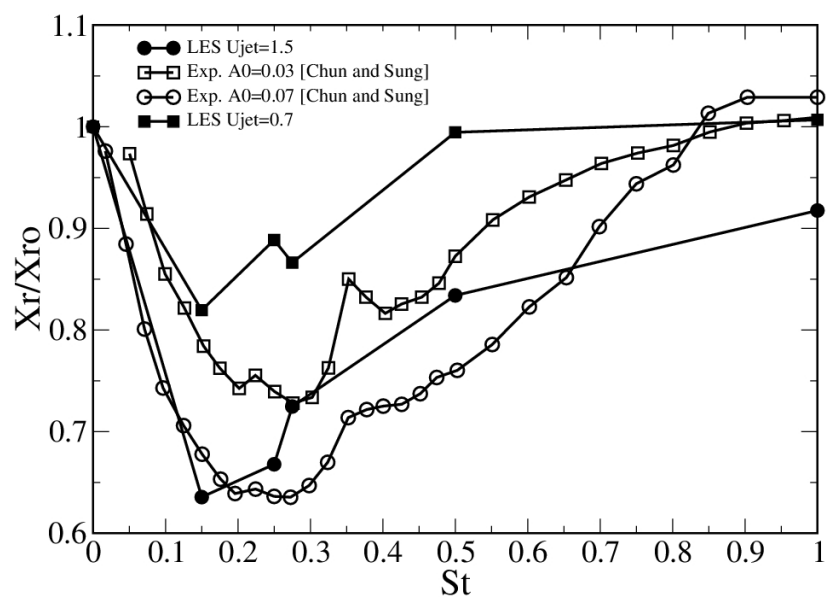

Figure 9: Ratio of the recirculation lengths of the controlled and natural flows vs the dimensionless micro-jet frequency, as obtained for two of the experiments of [23] and for the present simulations with $U_{\text {jet }}=1.5$ and $U_{\text {jet }}=0.7$.

More quantitatively, the influence of the micro-jet frequency on the recirculation length is shown in Fig. 9, which plots the ratio of the recirculation lengths of the controlled and natural flows versus the microjet Strouhal number $S t_{j e t}$. For the sake of comparison, some of the experimental results of [23] are also plotted. The experimental curves are those obtained for a difference between the natural and the controlled flow velocities of 0.05 and 0.07 at a measurement point close to the micro-jet aperture (parametrization used in [23]). These values roughly correspond to velocity amplitudes $U_{\text {jet }}$ between 0.7 and 1.5 in our computations. The experimental and numerical results, obtained for $U_{j e t}=0.7$ and $U_{\text {jet }}=1.5$, show a similar trend with a decrease of the recirculation length at the lowest frequencies. One observes however a slight shift of the minimum towards the low frequency and no increase of the recirculation length for $S t_{j e t}=1$ for the numerical results. One may think that a better agreement could be obtained with a longer time averaging. But it should also be recalled that the reattachment length is very sensitive to any changes in the flow, as e.g. highlighted by the comparison of experimental results of [26] (see Fig. 7), where the time averaging length is no-longer in question.

The influence of the synthetic micro-jet on statistic quantities is visualized in Fig. 10, which shows the mean streamwise velocity profile together with profiles of the Reynolds stresses $\left\langle u^{\prime} v^{\prime}\right\rangle$ and $\left\langle v^{\prime 2}\right\rangle$, as well as the profile of the turbulent kinetic energy $K$, at several streamwise locations. For the highest jet velocity $U_{j e t}=1.5$, one clearly observes the expected intensification of the momentum transfer across the shear layer, as e.g. discussed in $[27,31,26]$. Note however that our results are obtained at a higher Reynolds number and using at the upper boundary a free-slip condition rather than a no-slip one.

To conclude this Section, let us mention that some different control strategies may also be of interest 

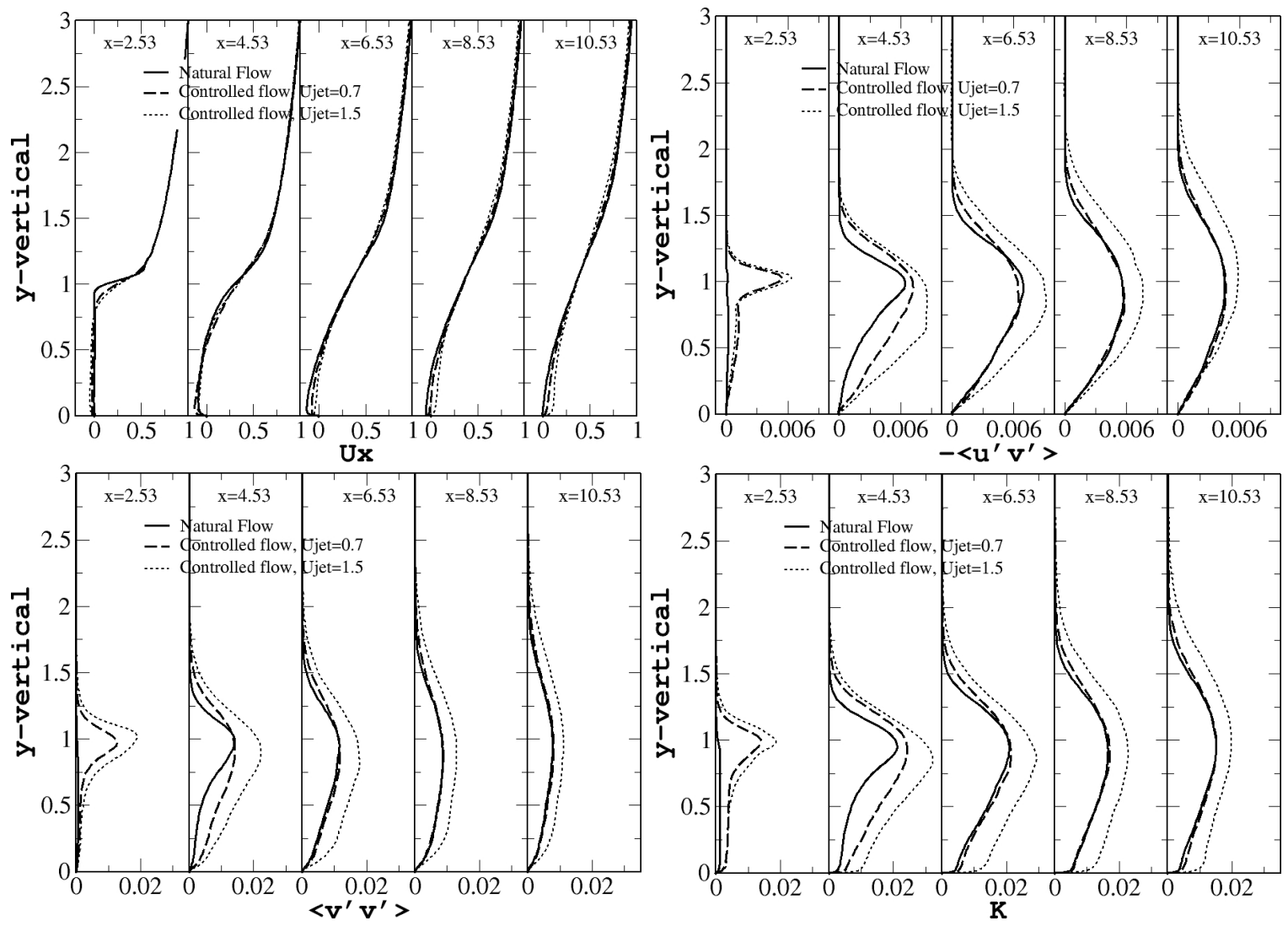

Figure 10: Profiles at several $x$-locations $\left(x=1.53\right.$ at the step) of $\left.\langle u\rangle\left(u \equiv u_{x}\right),-<u^{\prime} v^{\prime}\right\rangle,\left\langle v^{\prime 2}>\right.$ and $K=<\boldsymbol{u}^{\prime 2} / 2>$ for the natural and controlled flows with $S t_{\text {jet }}=0.15, U_{\text {jet }}=0.7$ and $U_{\text {jet }}=1.5$.

to modify the recirculation zone and the transport in the channel. Thus, in [32] two different techniques are investigated. The first one does not make use of micro-jets but is based on a pulsing inlet velocity. The second technique is based on a couple of micro-jets, both of them oriented in streamwise direction, one being located at the basis of the step and the second at the top. Different coupling strategies between the two micro-jets are then possible, with steady jets (one blowing and the other sucking) or with pulsing jets working in opposite phases. The 2D study that has been carried out shows that impressive results may thus be obtained, with diminution up to $50 \%$ of the recirculation length.

\subsection{D-shaped body}

The two-dimensional D-shaped bluff body is considered. This test case was investigated by several authors in numerical or experimental studies of wake flow control $[9,14,17,33]$. The height of the body, $h=$ $7.2 \times 10^{-2} \mathrm{~m}$, and the inlet velocity, $U_{i n}=4.17 \mathrm{~ms}^{-1}$, are used as reference length and velocity, respectively. The Reynolds number equals $R e=20000$ (the fluid is air). As in [14], the bluff body is located at a 
dimensionless distance 5.4375 from the inlet, the height of the channel equals 7.7 and its width equals 7.639. Then, the blockage factor, defined as the ratio between the bluff body section to the channel one, equals $12.99 \%$. In our computations, the length of the domain equals 16.3125, see Fig. 11.

At the top and at the bottom we use free-slip boundary conditions. At the inlet, a constant velocity profile of dimensionless value 1 is imposed. At the outlet, for the $\mathrm{OBC}$ we use an advection equation at velocity 1. No-slip conditions are considered at the bluff body, which is modelled using the pseudo-penalization technique, see Appendix A.

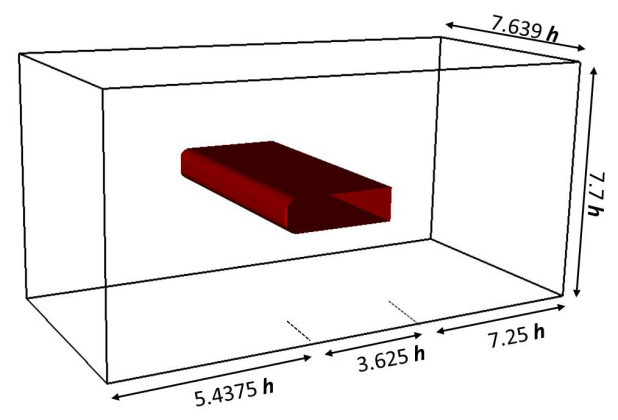

Figure 11: Sketch of the D-shaped bluff body.

The computational domain is composed of ten sub-domains in the $x$-streamwise direction. Three of them are located around the body, see Fig. 12(left). The front and rear parts of the body are located at two subdomain interfaces, in order to take advantage of the refinement of the Chebyshev mesh at the end-points. In each subdomain the polynomial approximation is $40 \times 254 \times 128$, yielding about 26.2 millions of gridpoints. In the $y$-transverse direction a mapping is used to accumulate grid-points at the top and bottom sides of the bluff body. In the $z$-spanwise direction the mesh is uniformly distributed. The dimensionless time-step was taken equal to $\tau=1.0 \times 10^{-3}$ (the reference time equals $h / U_{i n} \approx 1.73 \times 10^{-2} s$ ).

A snapshot of the streamwise velocity in the median $x-y$ plane together with the mesh is shown in Fig. 12(left). Based on the strategy of reducing the wake section to decrease the drag force, see e.g. [34], synthetic micro-jets are aligned at the rear of the body along the upper and lower edges. The inclination angle equals $45^{\circ}$ inwards in the $x-y$ plane, see Fig. 12(right) where a zoom of the upper edge is visualized. The pulsating frequency equals $S t_{j e t}=6.912$, which corresponds to $400 \mathrm{~Hz}$. The micro-jet intensity is characterized by the coefficient $C_{\mu}$, that we compute as suggested in [1], i.e. by taking its mean value during the expulsion phase of the micro-jet. When using dimensional values and assuming $u_{j e t}$ as positive during 

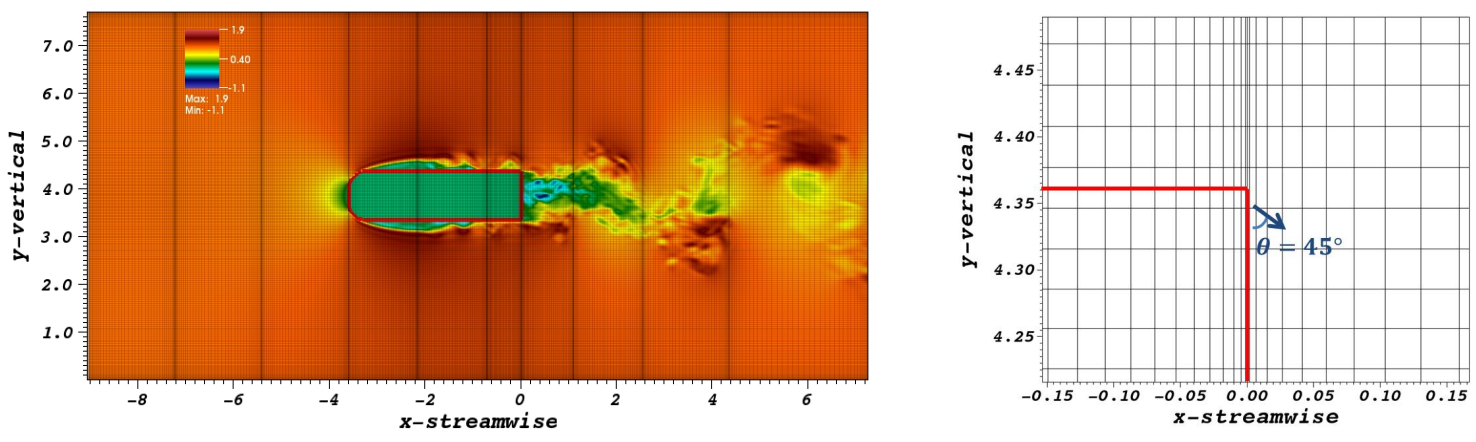

Figure 12: Instantaneous streamwise velocity and sketch of the computational grid (left). Micro-jets location in the rear upper part of the D-shaped body (right).

the expulsion phase:

$$
C_{\mu}=\frac{2 s_{j e t}<u_{j e t}^{2}>}{U_{i n}^{2} S}, \quad u_{j e t}>0
$$

where $s_{j e t}$ and $S$ are the micro-jet and bluff body sections. Then, with $u_{j e t}(t)=U_{j e t} \sin \left(2 \pi S t_{j e t} t\right)$, for the D-shaped body and in dimensionless form: $C_{\mu}=U_{j e t}^{2} s_{j e t}$, where $s_{j e t}$ is now the dimensionless thickness of the micro-jet.

\begin{tabular}{cc|cc|cc|cc|cc}
\hline \hline & & \multicolumn{2}{|c|}{$t \in(90,120)$} & \multicolumn{2}{c|}{$t \in(120,150)$} & \multicolumn{2}{c|}{$t \in(150,180)$} & \multicolumn{2}{c}{$t \in(90,180)$} \\
\hline Case & $C_{\mu}$ & $C_{d}$ & $\Delta C_{d} \%$ & $C_{d}$ & $\Delta C_{d} \%$ & $C_{d}$ & $\Delta C_{d} \%$ & $C_{d}$ & $\Delta C_{d} \%$ \\
\hline \hline DS0 & -- & 1.0628 & -- & 1.0380 & -- & 1.0487 & -- & 1.0498 & -- \\
\hline DS1 & 0.15 & 0.7348 & -30.86 & 0.7384 & -28.86 & 0.7343 & -29.98 & 0.7359 & -29.91 \\
\hline DS2 & 0.015 & 0.9908 & -6.77 & 0.9635 & -7.18 & 0.9341 & -10.93 & 0.9628 & -8.29 \\
\hline \hline
\end{tabular}

Table 1: Mean drag coefficient $C_{d}$ computed in the time interval $t \in(90,180)$.
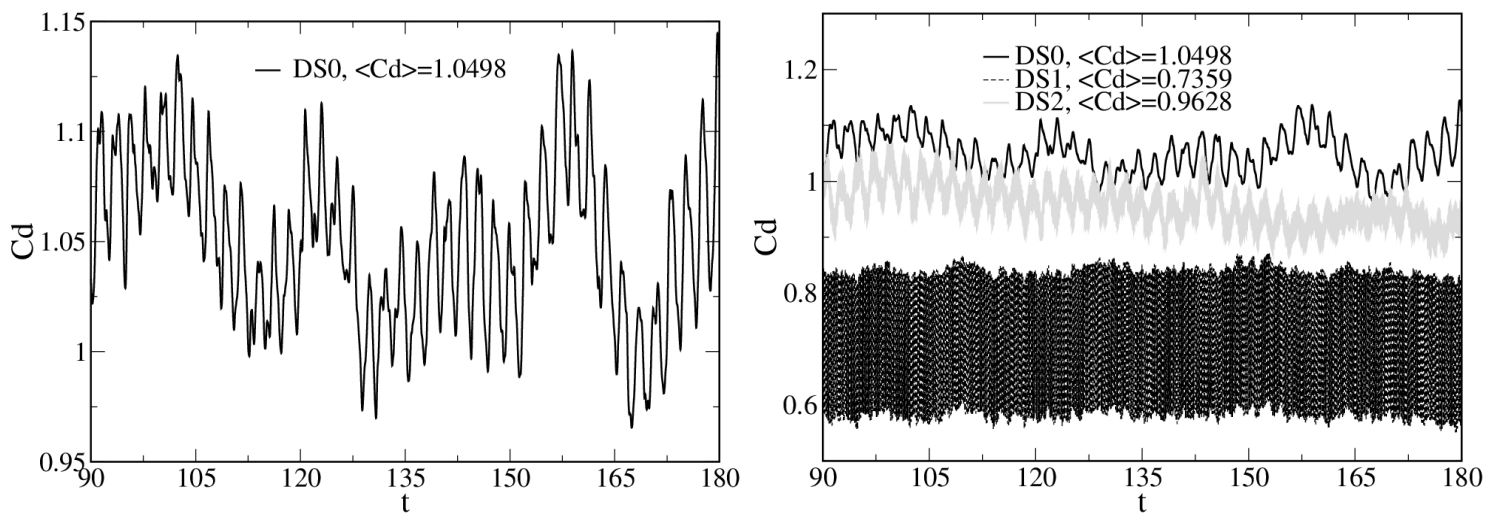

Figure 13: Time variation of the drag coefficient $C_{d}$ : Natural flow (left) and controlled flows (right).

Three cases have been computed, characterized by different values of the total $C_{\mu}$, see Table 1 . The 
case DS0 corresponds to the natural flow, whereas the cases DS1 to DS2 are for the controlled flows. Note that for all controlled flows, $U_{j e t}=1.5$, i.e. in Table 1 the variations of $C_{\mu}$ only result from variations of the jet section $s_{j e t}$. Table 1 also provides the drag coefficient $C_{d}$ obtained for the cases DS0 to DS2 as well as the gain that may be obtained with respect to the natural flow. In dimensionless form, here we simply have $C_{d}=2 F_{x}$, where $F_{x}$ is the $x$-component of the dimensionless force, say $\boldsymbol{F}$, applied by the flow onto the bluff body. Note that when using a penalization technique, the drag force may be computed by integrating the forcing term introduced inside the bluff body to (approximately) cancel the velocity. For the pseudo-penalization technique, details are provided in the Appendix A.
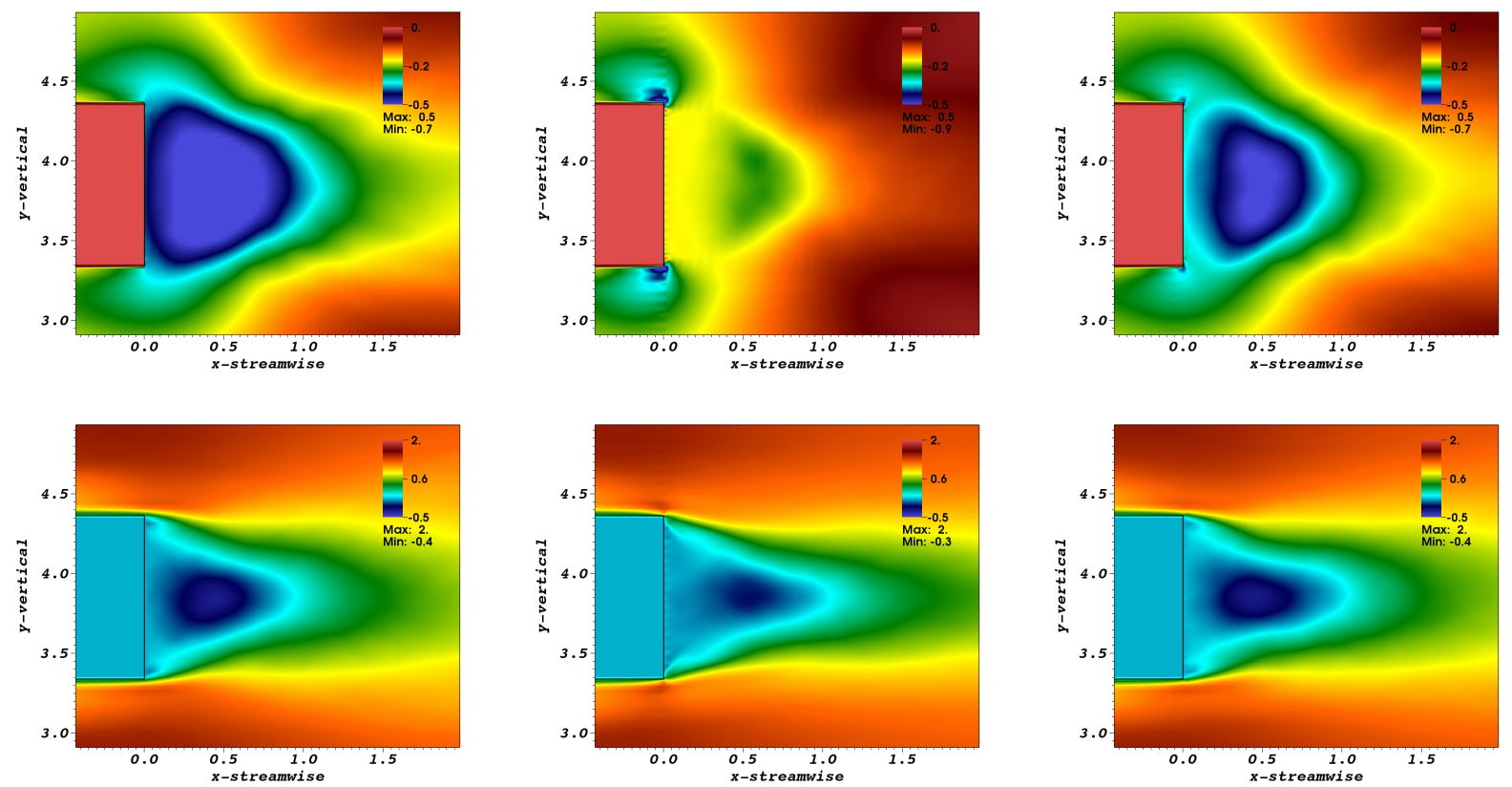

Figure 14: Mean Pressure field and streamwise velocity component $u \equiv u_{x}$ (from top to bottom) in the $z=0$ plane for the cases DS0 to DS2 (from left to right).

One notices that the value $C_{d}=1.05$ obtained for the natural flow, case DS0, falls within the range of values obtained numerically, $C_{d}=1.02$ in [14] and $C_{d}=1.14$ in [33], but slightly under-predicts the experimental results of [17], $C_{d}=1.17$. For the case DS1 an important diminution of the drag coefficient is obtained. This highlights the importance of using micro-jets of sufficiently high flow-rates. Figure 13 represents the time variations of $C_{d}$ for the cases DS0 (natural flow), DS1 and DS2 (controlled flow with $C_{\mu}=0.15$ and $C_{\mu}=0.015$ respectively).

To clearly understand the mechanism of drag reduction associated to the considered system, the effect of the micro-jets onto the flow is highlighted in figure 14, where the mean pressure field and the mean 
streamwise velocity component are presented for the three cases (DS0, DS1 and DS2). One observes that the introduction of micro-jets at the rear of the body leads to a confinement of the recirculation bubble and so to an increase of the pressure. More quantitatively, mean pressure profiles are presented for the different cases in figure 15, confirming the efficiency of the mechanism but again highlighting the importance of using micro-jets of sufficiently high flow-rates.
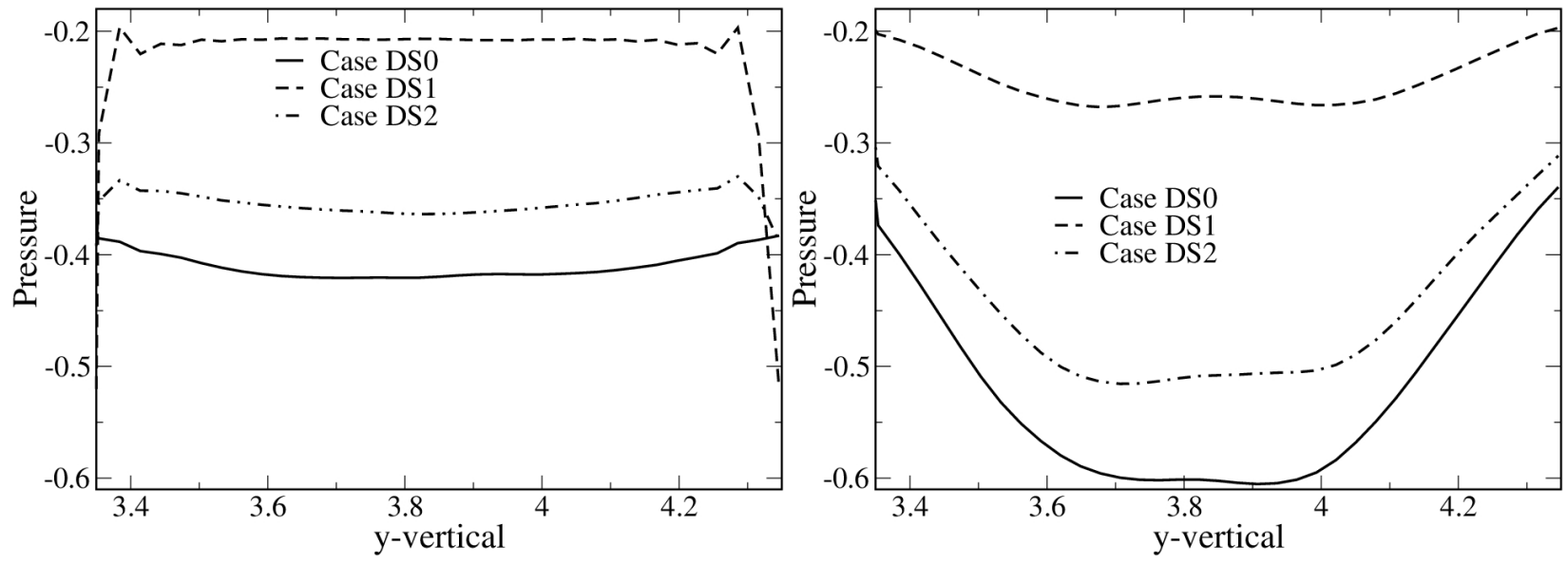

Figure 15: Mean Pressure transverse profiles in the $x=0.0067$ and $x=0.5$ planes at $z=0$ (from left to right).

\subsection{Ahmed body}

Since the pioneering work [35], the so called Ahmed body simplified car model has become a classical benchmark for numerical methods, turbulence modelling, experimental investigations and wake control. Concerning the numerical investigations, using LES or RANS approaches, see e.g. the ERCOFTAC report [36] or the DFG-CNRS review paper [37] and references herein for the Ahmed body with slant angle $30^{\circ}$. The experiments of [35] have shown that most of the drag is due to the pressure forces and that the largest contribution is associated to the near wake. Therefore it seems appropriate to use a strategy of drag reduction based on a perturbation of the near wake flow. For the square back Ahmed body, in [34] a reduction of the drag coefficient by means of blowing steady jets positioned around the rear of the body is obtained. In the frame of the ANR project LIVE-CAMS, one follows a similar strategy but using synthetic micro-jets.

The Reynolds number, based on the height $h=0.288 \mathrm{~m}$ of the body (of width, $0.389 \mathrm{~m}$ and length $1.044 \mathrm{~m}$ ) and on the mean inlet velocity $U_{i n}=26.7 \mathrm{~ms}^{-1}$ equals $R e=512000$. Computations are carried out in a channel like geometry of dimensionless length 16.3125, height 3.2 and width 4.32, see Fig. 16(left). The bluff body is located at 5.4375 from the inlet. The blockage factor, defined as the ratio between the bluff body section to the channel one, equals $9.77 \%$. A free slip condition is used at the top of the domain, 

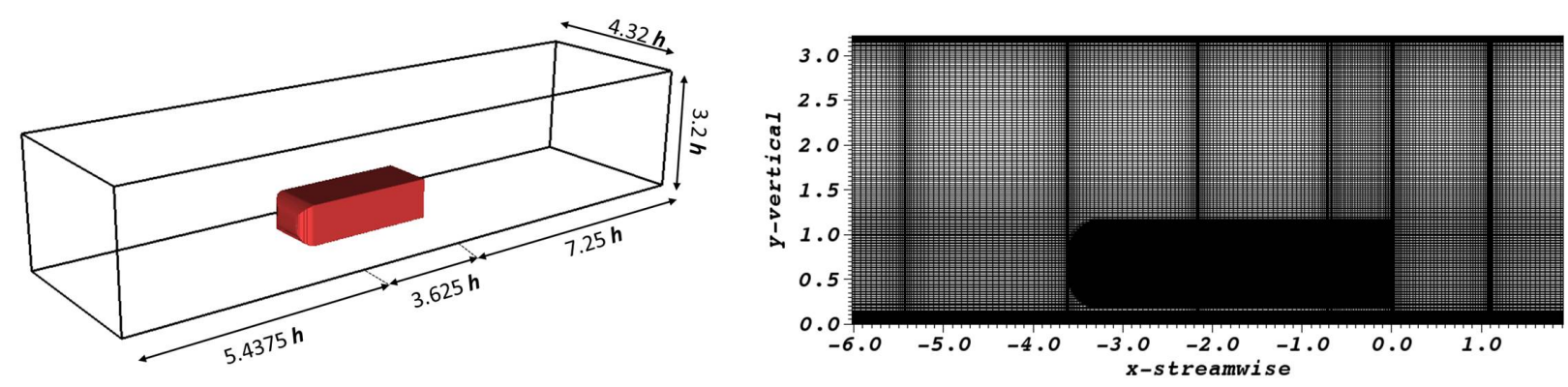

Figure 16: Computational domain and mesh.

a no-slip condition is used at the ground, at the inlet one uses a boundary layer profile and at the outlet an advection equation for OBC. No-slip conditions are considered at the Ahmed body, using again the pseudo-penalization technique.

The computational domain is composed of ten sub-domains in the $x$-streamwise direction. Three are located around the Ahmed body as sketched in Fig. 16(right). In each subdomain the polynomial approximation is $40 \times 190 \times 128$, yielding about 19.6 millions of grid-points. In the $y$-vertical direction a mapping is used to accumulate grid-points at the roof of the car model. In the $z$-spanwise direction the mesh is uniformly distributed. The dimensionless time-step was taken equal to $\tau=2.0 \times 10^{-3}$. The reference time equals $h / U_{\text {in }} \approx 1.08 \times 10^{-2} s$.

Results for the natural flow are provided in Fig. 17, which shows the instantaneous and mean streamwise velocity components (lines 1 and 2), the instantaneous and mean pressure fields (lines 3 and 4) in the $z=0$ and $y=0.67$ planes and the turbulent kinetic energy in the same planes (line 5). The mean quantity results show a lack of symmetry that can be attributed to the bi-modality phenomenon, with alternatively two different configurations of the flow $[38,39]$. Our simulation time is indeed much too short to recover symmetric statistics with respect to the symmetry plane of the Ahmed body. As expected, a high turbulence activity is present in the shear layers around the recirculation zones.

Figure 18 compares profiles of the time-averaged streamwise velocity component, $u_{x}$, with the experimental results of [39]. The experimental results have been obtained for $R e=300000$ and with an Ahmed body slightly different from the original one, i.e. of length $0.893 \mathrm{~m}$, width $0.35 \mathrm{~m}$ and height $0.297 \mathrm{~m}$. When taking into account such differences, the agreement may be considered reasonably satisfactory.

From comparisons with the experiments presently carried in the frame of LIVE-CAMS it turns out that [40]:

- the topology of the flow in the near wake is satisfactory, especially the recirculation behind the Ahmed 

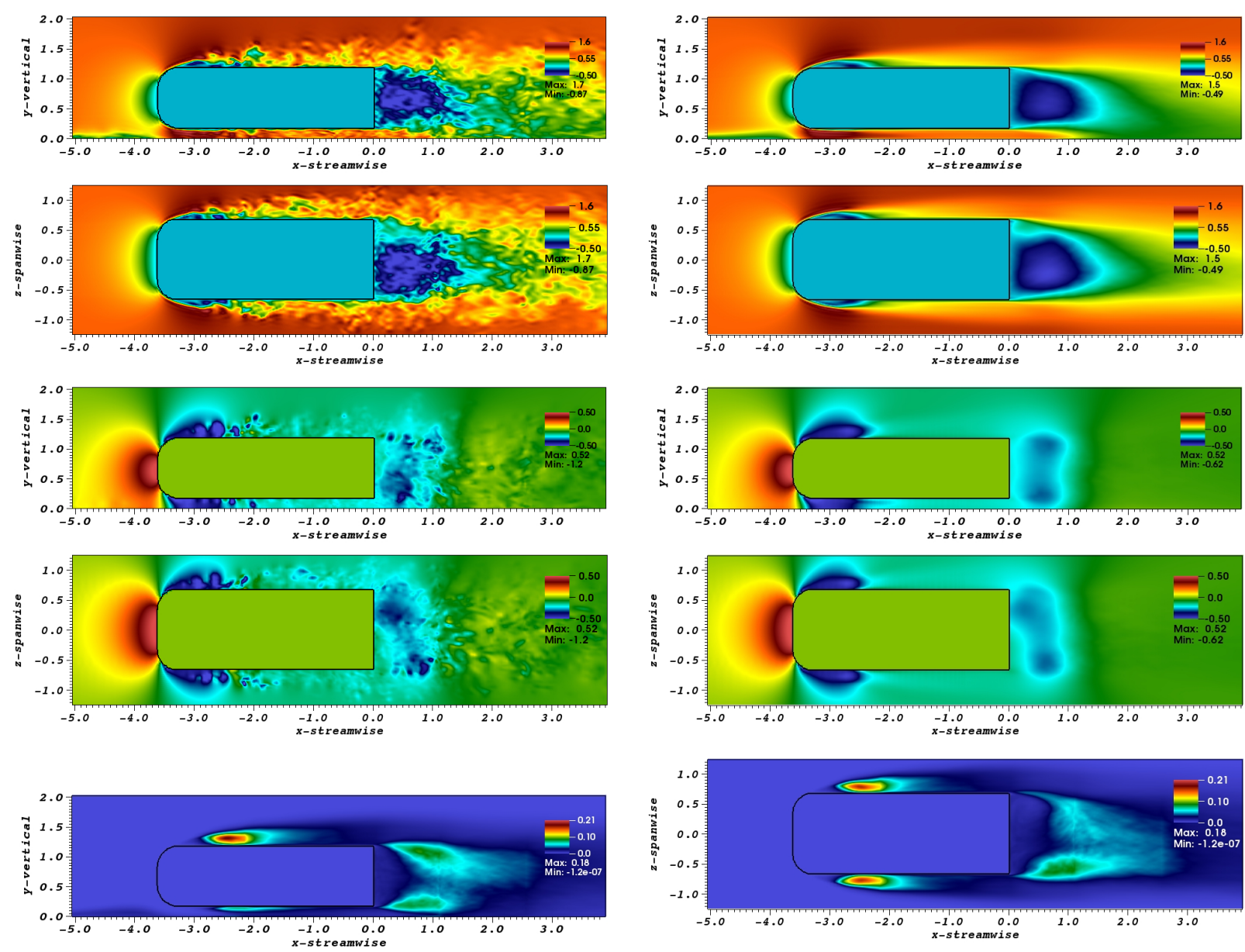

Figure 17: Lines 1 and 2: Instantaneous and mean streamwise velocity component (from left to right) in the $z=0$ and $y=0.67$ planes (from top to bottom). Lines 3 and 4: Corresponding pressure fields. Line 5: Turbulent kinetic energy in the $z=0$ and $y=0.67$ planes (from left to right)

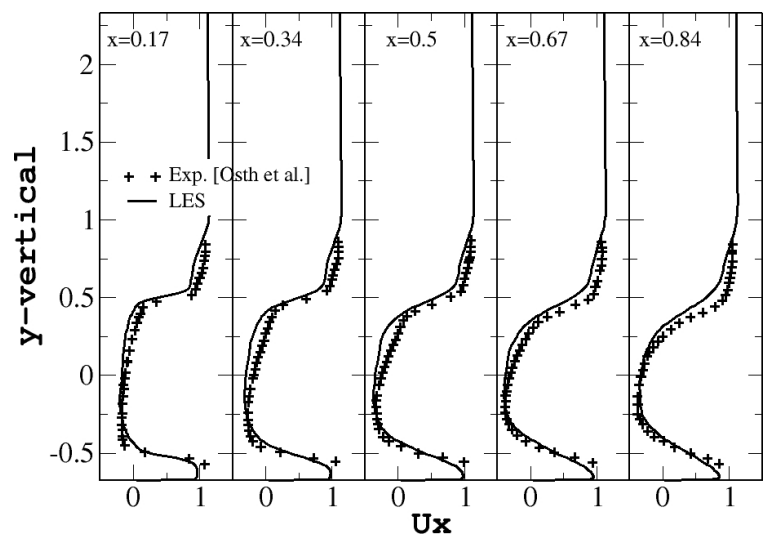

Figure 18: Mean streamwise velocity profiles in the median vertical plane at different $x$-locations with comparison to the experimental data of [39]. For this comparison, the $y$-axis is such that at the back of the Ahmed body: $-0.5<y<0.5$. 
body is well described.

- The recirculation zones in the front part of the Ahmed body are not observed in the experiments. This may be attributed to the fact that the mesh used in the present study does not allow to resolve the boundary layers. Experimentally, such recirculations are indeed generally obtained at lower Reynolds numbers. As discussed hereafter, this may also result from the channel like geometry of the computational domain.

- The drag coefficient is over-predicted in the simulations. We indeed find $C_{d} \approx 0.65$ to be compared to the experimental value $C_{d} \approx 0.32$. The pressure force contribution being very dominant, this cannot be attributed to excessive viscous effects. As argued in [41], where an over-prediction by a factor of 2 is also observed, this is actually associated to the fact that the fluid cannot leave the computational domain through the side and top boundaries. In [41], where the blocking factor (ratio of the Ahmed body and computational domain sections) equals $12.47 \%$, this was cured by using at these boundaries a constant pressure boundary condition. Such a boundary condition cannot be easily implemented in the SVVLES spectral solver. Thus, the present drag coefficient corresponds more to the one obtained inside a tunnel than in a semi-open medium. This was not the case for the D-shaped body, where the comparisons with both the experiments and other numerical simulations were satisfactory, since indeed both of them were carried out in a channel.
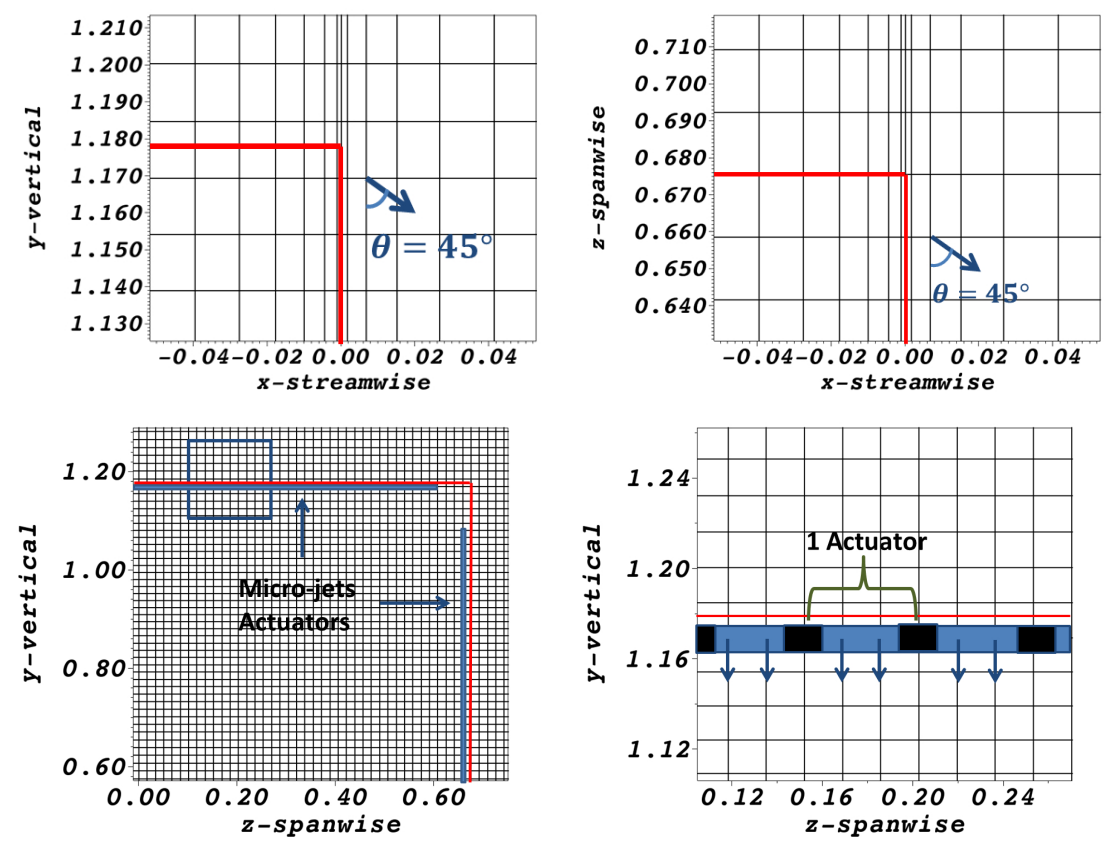

Figure 19: Jet locations in the $z=0$ (top left), $y=0.67$ (top right) and $x=0.0067$ planes (bottom left). The angle between each jet and the wall is $\theta=45^{\circ}$ inward. Details of the micro-jet modelling (bottom right). 


\begin{tabular}{llllllll}
\hline \hline Case & $\begin{array}{l}\text { Frequency } \\
(\mathrm{Hz})\end{array}$ & $\begin{array}{l}\text { Velocity max } \\
(\mathrm{m} / \mathrm{s})\end{array}$ & $\begin{array}{l}\text { Section } \\
\left(\mathrm{mm}^{2}\right)\end{array}$ & $\begin{array}{l}\text { Number } \\
\text { (hor.-ver. })\end{array}$ & $C_{\mu}$ & $\begin{array}{l}\text { Inclination } \\
\left({ }^{\circ}\right)\end{array}$ & $\begin{array}{l}\text { Lagging } \\
\text { phase }\end{array}$ \\
\hline \hline AB0 & --- & --- & --- & $0-0$ & --- & --- & --- \\
\hline AB1 & 800 & 40 & $10 \times 2$ & $24-0$ & $9.65 \times 10^{-3}$ & 45 & No \\
\hline AB2 & 300 & 40 & $10 \times 2$ & $24-18$ & $1.69 \times 10^{-2}$ & 45 & No \\
\hline AB3 & 800 & 40 & $10 \times 0.2$ & $24-18$ & $1.69 \times 10^{-3}$ & 45 & No \\
\hline AB4 & 800 & 40 & $10 \times 2$ & $24-18$ & $1.69 \times 10^{-2}$ & 45 & No \\
\hline AB5 & 800 & 40 & $10 \times 2$ & $24-18$ & $1.69 \times 10^{-2}$ & 0 & No \\
\hline AB6 & 800 & 40 & $10 \times 2$ & $24-18$ & $1.69 \times 10^{-2}$ & 45 & yes \\
\hline \hline
\end{tabular}

Table 2: Test-cases for the Ahmed body car model.

Concerning the controlled flows, the micro-jets are located at the rear of the Ahmed body and along its four edges. To model these micro-jets, here again one uses punctual forcing terms, located at two grid-points away from the walls, see Fig. 19. Moreover, on the basis of the studies carried out in the frame of LIVECAMS, at the rear of the body the grid-size is chosen such that 3 grid-spaces correspond to each actuator and two of them are associated to the jet itself. Then one may have 24 actuators along the horizontal edges and 18 along the vertical ones. Several test-cases, characterized by the number, inclination and frequency of the micro-jets, as summarized in Table 2, have been investigated. Concerning the case AB6, side by side micro-jets work in opposite expulsion-ingestion phases.

\begin{tabular}{c|cc|cc|cc|cc}
\hline \hline & \multicolumn{2}{|c|}{$t \in(80,180)$} & \multicolumn{2}{|c|}{$t \in(180,280)$} & \multicolumn{2}{c|}{$t \in(280,380)$} & \multicolumn{2}{c}{$t \in(80,380)$} \\
\hline Case & $C_{d}$ & $\Delta C_{d} \%$ & $C_{d}$ & $\Delta C_{d} \%$ & $C_{d}$ & $\Delta C_{d} \%$ & $C_{d}$ & $\Delta C_{d} \%$ \\
\hline \hline AB0 & 0.6558 & -- & 0.6556 & -- & 0.6585 & -- & 0.6566 & -- \\
\hline AB1 & 0.6462 & -1.47 & 0.6497 & -0.90 & 0.6415 & -2.57 & 0.6458 & -1.65 \\
\hline AB2 & 0.6384 & -2.65 & 0.6423 & -2.04 & 0.6363 & -3.36 & 0.6390 & -2.68 \\
\hline AB3 & 0.6463 & -1.45 & 0.6470 & -1.31 & 0.6477 & -1.64 & 0.6470 & -1.47 \\
\hline AB4 & 0.6251 & -4.69 & 0.6326 & -3.52 & 0.6287 & -4.52 & 0.6288 & -4.24 \\
\hline AB5 & 0.6569 & +0.17 & 0.6526 & -0.46 & 0.6525 & -0.91 & 0.6540 & -0.40 \\
\hline AB6 & 0.6293 & -4.05 & 0.6319 & -3.62 & 0.6340 & -3.71 & 0.6317 & -3.79 \\
\hline \hline
\end{tabular}

Table 3: Mean drag coefficient $C_{d}$ computed in the time interval $t \in(80,380)$.

Table 3 presents the results obtained for the drag coefficient $C_{d}$. The statistics have been computed over 300 time units, but we also provide the results associated to three successive time intervals of 100 time units. The best result is obtained for the case AB4, i.e. with inclined micro-jets working at high frequency, with a drag coefficient reduction of $4.24 \%$. By comparing the cases AB4 and AB5, one observes that it is important to use inclined rather than horizontal micro-jets. Also, from the comparison of cases AB2 and $\mathrm{AB} 4$, one observes that using high frequency micro-jets is desirable. As stated in section 4.2, the results also highlight the importance of using a system with high $C_{\mu}$ : When decreasing the micro-jet number (case AB1) or section (case AB3), worse results are indeed obtained. Finally, it does not seem important here to use side by side micro-jets working in opposite phase, case AB6 with respect to AB4. 

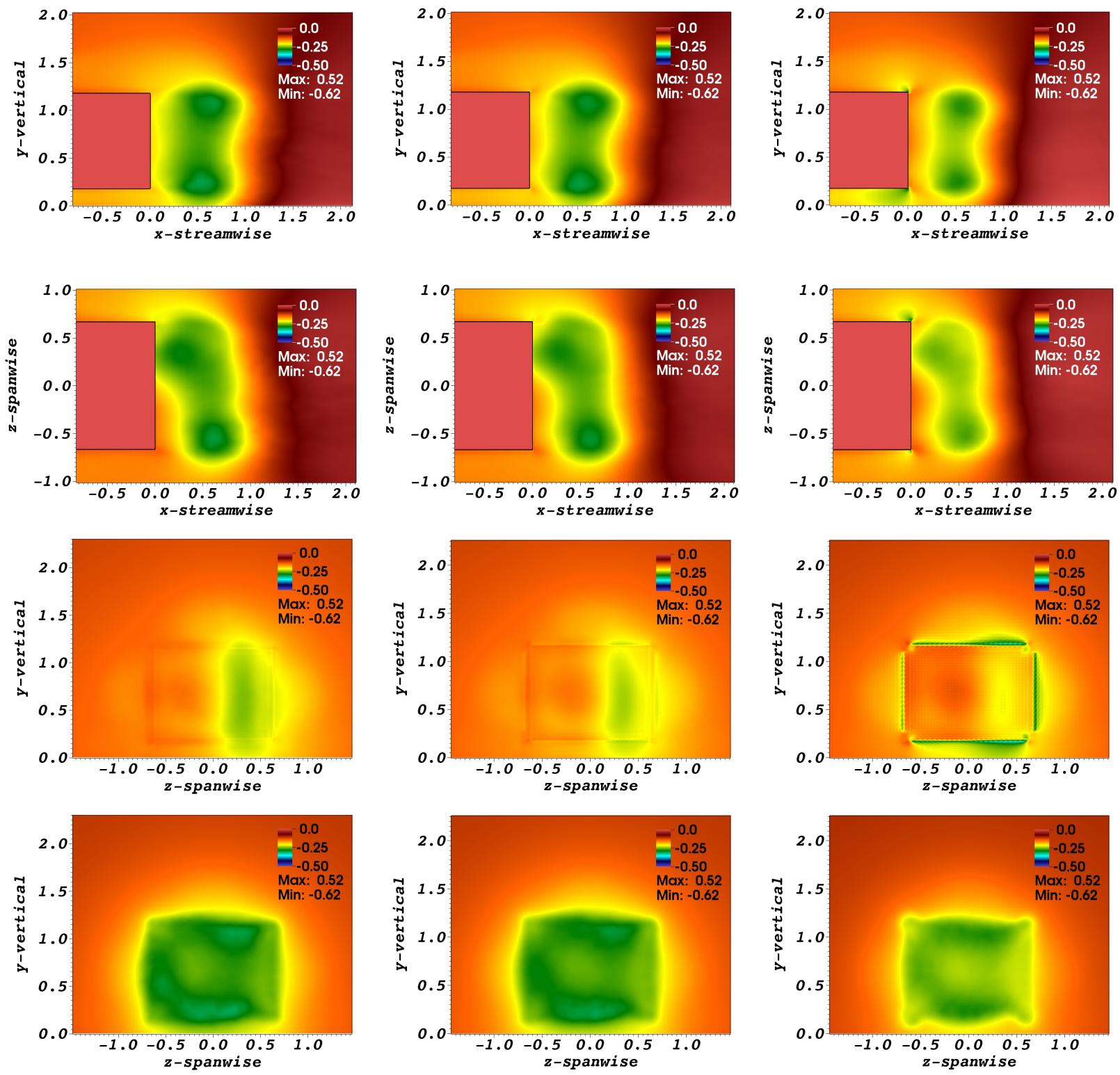

Figure 20: Mean pressure field for the cases AB0, AB3 and AB4 (from left to right) in the in the planes: $z=0$ (first line), $y=0.67$ (second line), $x=0.0067$ (third line) and $x=0.5$ (fourth line).

The mean pressure fields obtained for the cases $A B 0$ (natural flow), $A B 3$ and $A B 4$ (controlled flow with $C_{\mu}=1.69 \times 10^{-3}$ and $C_{\mu}=1.69 \times 10^{-2}$ respectively) are compared in Fig. 20. The results obtained for the case $\mathrm{AB} 4$ are in line with those presented for the D-shaped geometry, case DS2 with a greater $C_{\mu}$. As expected, the strategy adopted to control the wake flow leads to a decrease of the wake cross section as well as an increase of the pressure. More quantitavely, mean pressure profiles at two streamwise locations are presented in figure 21. 

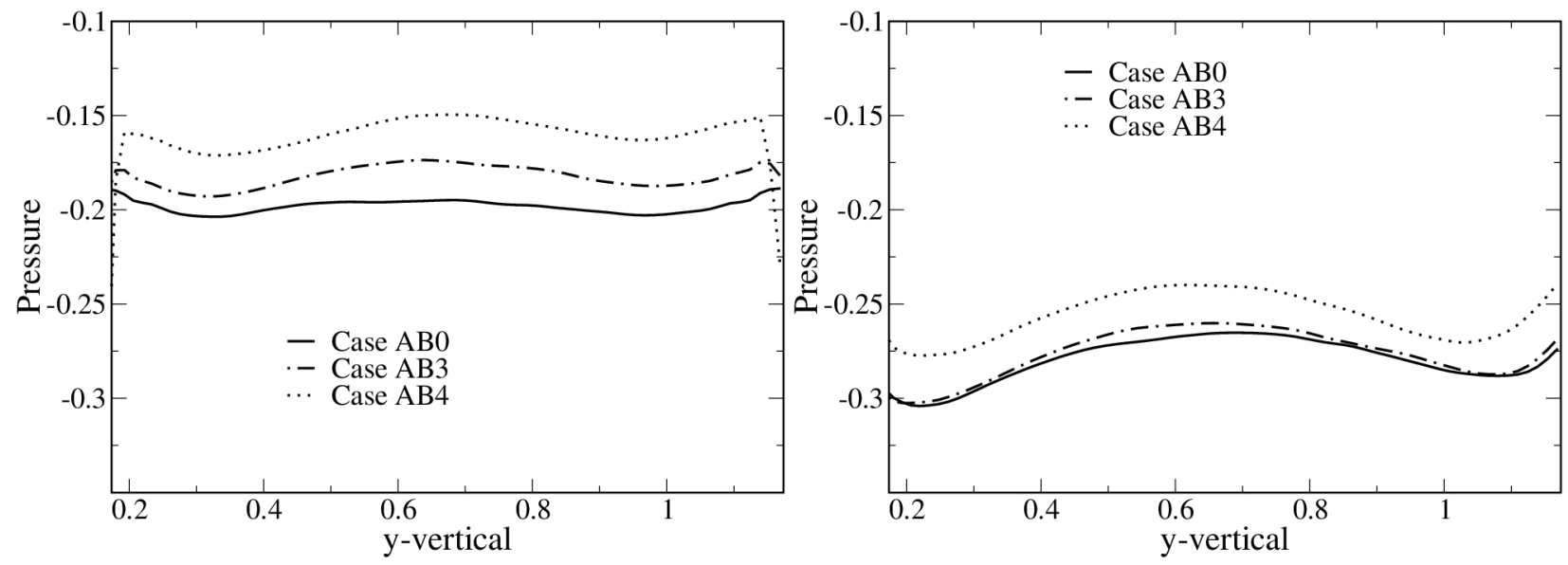

Figure 21: Mean Pressure vertical profiles in the $x=0.0067$ and $x=0.5$ planes at $z=0$ (from left to right).

To conclude this Section, let us mention that comparisons to the experiments carried out in the frame of the ANR project LIVE-CAMS together with a more detailed discussion of the physical phenomena, will be provided in a forthcoming paper. Moreover, an interesting analysis of the controlled wake of the back square Ahmed body, using various control strategy, may already be found in the recent $\mathrm{PhD}$ work [42].

\section{Concluding remarks}

When complex real life flows are concerned, taking into account the presence of micro-jets immediately leads to a challenging multi-scale problem. To face such problems, a modelling of the micro-jet is required and the goal of the present paper was then to propose a simple model of synthetic micro-jets. Starting from the conservation equations of mass and momentum, we have shown that the micro-jet could be reasonably modelled by introducing in the momentum conservation equation a penalty like term, with a time-dependent penalty coefficient that culminates in the expulsion phase and vanishes in the ingestion one. Up to our knowledge, such a model of synthetic micro-jet is new. We have shown that it could be easily implemented in a Fourier-Chebyshev spectral code (SVVLES code) and tested against a detailed computation of the flow induced by a single micro-jet in a fluid at rest. Finally, the interest of the proposed modelling has been pointed out by considering different 3D test cases involving turbulent flows: the backward facing step, for which the experimental trends have been recovered, the D-shaped body and the Ahmed body.

In case of the D-shaped and Ahmed bodies, the strategy to reduce the drag force was based on the use of micro-jets located at the rear and acting on the near wake, in order to reduce the recirculation zone and increase the pressure level. It turns out that such a strategy requires micro-jets of high intensity (high $C_{\mu}$ ). Thus, for the D-shaped body, see Section 4.2, case DS1, it appears possible to get a drag reduction around 
$30 \%$, but with $C_{\mu}=0.15$. Similarly, for the square back Ahmed body, the best result, case AB4, has been obtained for $C_{\mu} \approx 0.017$. The question that naturally arises is then to know if the energy balance remains favourable. Although investigating this crucial point is out of the scope of the present paper, some guidelines are provided in Appendix B.

\section{Acknowledgments}

This work was supported by the French National Research Agency (ANR), in the frame of the project Limitation of the impact of vehicles on the environment by means of aerodynamic control using synthetic micro-jets (LIVE-CAMS). We thank all the partners of this project for fruitful discussions. The calculations were mainly carried out on the cluster of the Centre de Calculs Interactifs of the University of Nice Sophia Antipolis.

\section{Appendix A. Pseudo-penalization technique}

In order to enforce the velocity $\boldsymbol{u}=\boldsymbol{u}_{\text {obst }}$ in the obstacle, defined by the domain $D_{\text {obst }}$, we use the pseudopenalization technique. Since at each time step the predicted velocity $\boldsymbol{u}^{\star}$ that results from the diffusion step solves an equation of the form:

$$
\nu \Delta \boldsymbol{u}^{\star}-\frac{\alpha}{\tau} \boldsymbol{u}^{\star}=-\nabla p^{\star}+\boldsymbol{f}
$$

where $\boldsymbol{f}$ and $p^{\star}$ are scheme dependent source terms, the pseudo-penalization technique consists in solving

$$
\nu \Delta \boldsymbol{u}^{\star}-\frac{\alpha}{\tau} \boldsymbol{u}^{\star}=-\nabla p^{\star}+\left(1-\chi_{o b s t}\right) \boldsymbol{f}-\chi_{o b s t} \frac{\alpha}{\tau} \boldsymbol{u}_{o b s t}
$$

so that in the obstacle, of characteristic function $\chi_{\text {obst }}$, arises a penalized steady Stokes problem with penalty term $\alpha\left(\boldsymbol{u}^{\star}-\boldsymbol{u}_{\text {obst }}\right) / \tau$. Thus, the penalization term introduced by the pseudo-penalization technique is of order $O(1 / \tau)$. Of course, if the obstacle is at rest $\boldsymbol{u}_{\text {obst }}=0$ and the pseudo-penalization technique then simply consists of canceling the source term $f$ inside the obstacle.

Now, to explain how we compute the drag force of the obstacle, let us restart from the momentum conservation equation with forcing term $\boldsymbol{F} \neq 0$ in the obstacle

$$
\partial_{t}(\rho \boldsymbol{u})+\nabla \cdot(\rho \boldsymbol{u} \boldsymbol{u}+P \mathbb{I}-\sigma)=\boldsymbol{F}
$$


and recall that the resulting force on the obstacle is

$$
\int_{\Gamma_{\text {obst }}}(-P \mathbb{I}+\sigma) \cdot d \boldsymbol{\Gamma}
$$

Then, by integrating on $D_{\text {obst }}$ and applying to $\nabla \cdot(P \mathbb{I}-\sigma)$ the divergence theorem,

$$
\boldsymbol{R}=\int_{D_{\text {obst }}}\left(\partial_{t}(\rho \boldsymbol{u})+\nabla \cdot(\rho \boldsymbol{u u})-\boldsymbol{F}\right) d D
$$

The time derivative is now approximated with a backward finite difference formula and the non-linear term is treated explicitly. Then we are left at time $t_{n+1}$ with

$$
\boldsymbol{R}=\int_{D_{\text {obst }}}\left(\frac{\alpha}{\tau}(\rho \boldsymbol{u})^{n+1}+\boldsymbol{f}-\boldsymbol{F}\right) d D
$$

where $f$ depends on the previous times $t_{n}, t_{n-1}, \cdots$. Clearly, in the frame of the pseudo-penalization technique one sets $\boldsymbol{F}=\boldsymbol{f}$ in the obstacle, so that:

$$
\boldsymbol{R}=\int_{D_{o b s t}} \frac{\alpha}{\tau}(\rho \boldsymbol{u})^{n+1} d D
$$

The drag and lift coefficients are obtained by using the reference quantity $\rho S U^{2} / 2$, where $S$ is the bluff body section and $U$ the upstream velocity. For $\rho$ constant and if dimensionless values are used, so that $U=1$, one obtains the dimensionless resulting force

$$
\boldsymbol{C}\left(t_{n+1}\right)=\frac{2}{S} \int_{D_{\text {obst }}} \frac{\alpha}{\tau} \boldsymbol{u}^{n+1} d D
$$

The pressure contribution, say $\boldsymbol{C}_{p}$, is easy to compute:

$$
\boldsymbol{C}_{p}\left(t_{n+1}\right)=-\frac{2}{S} \int_{\Gamma} p^{n+1} d \boldsymbol{\Gamma}=-\frac{2}{S} \int_{D_{\text {obst }}} \nabla p^{n+1} d D
$$

where again $p=P / \rho$. In practice, all integrations in $D_{\text {obst }}$ are actually made in the computational domain $\Omega$ after multiplication of the kernel by $\chi_{o b s t}$. 


\section{Appendix B. Energy efficiency}

On the one hand, a decrease $\delta F_{x}$ for a bluff body moving at velocity $U$, is associated to a gain of kinetic power $\delta P_{g}=\delta F_{x} U$. Since $C_{d}=2 F_{x} /\left(\rho S U^{2}\right)$, one has $\delta P_{g}=\delta C_{d} \rho S U^{3} / 2$.

On the other hand, for steady micro-jets (in this case, $u_{j e t}(t)=U_{j e t}$ ), the power required is at least $P_{j e t}=\rho U_{j e t}^{2} s_{j e t} U_{j e t} / 2$, where $s_{j e t}$ stands for the sum of the micro-jets sections, to bring a fluid at rest to the velocity $U_{j e t}$.

Clearly, one must have $P_{j e t} \ll \delta P_{g}$, which means:

$$
\begin{aligned}
\frac{1}{2} \rho U_{j e t}^{2} s_{j e t} U_{j e t} & \ll \delta C_{d} \rho S U^{3} \\
\frac{U_{j e t}^{2} s_{j e t}}{0.5 U^{2} S} \frac{U_{j e t}}{4 U} & \ll \delta C_{d} \\
C_{\mu} & \ll \delta C_{d} \frac{4 U}{U_{j e t}} .
\end{aligned}
$$

A similar computation can be carried out in case of synthetic micro-jets: The power required by the micro-jet is at least:

$$
P_{j e t}=\frac{1}{T} \int_{0}^{T} \frac{1}{2} \rho u_{j e t}^{2} s_{j e t} u_{j e t}^{+} d t
$$

where $T=1 / S t_{j e t}$ is the time-period and with $u_{j e t}^{+}=\max \left(u_{j e t}, 0\right)$, because we assume that energy is only needed in the expulsion phase (again it is supposed that the micro-jet is passive in the ingestion one).

If as previously we assume that $u_{j e t}(t)=U_{j e t} \sin (2 \pi t / T)$, a simple calculation yields $P_{j e t}=2 s_{j e t} \rho U_{j e t}^{3} / 3$. Then we have:

$$
\begin{aligned}
\frac{2}{3} \rho s_{j e t} U_{j e t}^{3} & \ll \delta C_{d} \rho S U^{3} \\
\frac{U_{j e t}^{2} s_{j e t}}{U^{2} S} \frac{2 U_{j e t}}{3 U} & \ll \delta C_{d} \\
C_{\mu} & \ll \delta C_{d} \frac{3 U}{2 U_{j e t}} .
\end{aligned}
$$

where, as suggested in [1], $C_{\mu}$ is here only associated to the expulsion phase. In several examples of the paper, we have $U_{j e t} / U=1.5$, then one simply gets $C_{\mu} \ll \delta C_{d}$.

Such an analysis is of course optimistic, since the energy losses associated to the efficiency of the micro-jet were not taken into account. 


\section{References}

[1] A. Glezer, M. Amitay, Synthetic jets, Annu. Rev. Fluid Mech. 34 (2002) 503-529.

[2] D. Greenblatt, I. J. Wygnanski, The control of flow separation by periodic excitation, Progress in Aerospace Sciences 36 (2000) 487-545.

[3] J. C. Gerbedoen, P. Pernod, A. Talbi, Private communication, ANR project LIVE-CAMS, 2013.

[4] D. Lockerby, P. Carpenter, C. Davies, Numerical simulation of the interaction of microactuactors and boundary layers, Vol. 40(1), 2002, pp. 67-73.

[5] S. G. Mallinson, C. Y. Kwork, J. A. Reizes, Numerical simulation of micro-fabricated zero mass-flux jet actuators, Sensor and Actuators A 105 (2002) 229-236.

[6] C. Nae, Numerical simulation of a synthetic jet actuator, in: 22nd International Congress of Aeronautical Sciences, ICAS 2000 Proc., 266.1-266.9, 2000.

[7] C. Rumsey, T. Gatsky, W. sellers, V. Vatsa, S. Viken, Summary of the 2004 CFD validation workshop on synthetic jets and turbulent separation control, Vol. AIAA paper-2004-2217, 2004.

[8] R. Raju, E. Aram, R. Mittal, L. Cattafesta, Simple models of zero-net mass flux jets for flow control simulations, Int. J. of flow control 1 (3) (2009) 179-197.

[9] C. Bruneau, E. Creusé, D. Depeyras, I. Mortazavi, P. Gilliéron, Coupling passive and active techniques to control the flow past the square back Ahmed body, Comp. \& Fluids 38 (2010) 1875-1892.

[10] L. Kraal, J. Donovan, A. Cain, A. Carry, Numerical simulation of synthetic jets actuators, Vol. AIAA paper $97-1824,1997$.

[11] C. Lee, D. Goldstein, DNS of microjets for turbulent boundary layer control, Vol. AIAA paper-1013, 2001.

[12] K. A. Waithe, Source term model for steady micro jets in a Navier-Stokes computer code, Vol. AIAA paper-1401, 2005.

[13] Y. Eulalie, I. Mortazavi, P. Gilotte, P. Bobillier, Wake analysis and drag reduction for a square back ahmed body using LES computations, in: ASME 2014 4th Joint US-European Fluids Engineering Division Summer Meeting, Chicago, Illinois, USA, August 3-7, 2014, pp. Paper No. FEDSM2014-21552, pp. V01CT17A010; 7 pages. 
[14] S. Krajnovic, J. Fernandes, Numerical simulation of the flow around a simplified vehicle model with active flow control, Int. J. of Heat and Fluid Flow 32 (2011) 192-200.

[15] P. Joseph, X. Amandolèse, J. Aider, Drag reduction of the $25^{0}$ slant angle Ahmed reference body using pulsed jets, Exp. Fluids 52 (2012) 1169-1185.

[16] A. Kourta, C. Leclerc, Characterization of synthetic jet actuation with application to Ahmed body wake, Sensors and Actuators A 192 (2013) 13-26.

[17] M. Pastoor, L. Henning, B. R. Noack, R. King, G. Tadmor, Feedbac shear layer control for bluff body drag reduction, J. Fluid Mech. 608 (2008) 161-196.

[18] A. Thacker, S. Aubrun-Sanches, A. Leroy-Chesneau, P. Devinant, Effects of suppressing the 3D separation on the rear slant on the flow structures around an Ahmed body, J. Wind Eng. Ind. Aerodyn. (2012) 237-243.

[19] N. Peres, R. Pasquetti, Numerical study of drag reduction of a car model by active control using microjets, in: Proc. of the CFM 2013 Congress, August 26-30, 2013, Bordeaux, France.

[20] N. Peres, R. Pasquetti, SVV-LES of flow around the square-back Ahmed body, in: Proc. of the DLES 9 workshop, April 3-5, 2013, Dresden, Germany.

[21] M. Minguez, R. Pasquetti, E. Serre, Spectral vanishing viscosity stabilized LES of the Ahmed body turbulent wake, Commun. Comput. Phys. 5 (2009) 635-648.

[22] R. Pasquetti, R. Bwemba, L. Cousin, A pseudo-penalization method for high Reynolds number unsteady flows, Applied Numerical Mathematics 58 (2008) 946-954.

[23] K. B. Chun, H. J. Sung, Control of turbulent separated flow over a backward-facing step by local forcing, Experiments in fluids 21 (1996) 417-426.

[24] S. Yoshioka, S. Obi, S. Masuda, Momentum transfer in the periodically perturbed turbulent separated flow over the backward-facing step, in: Proc. TSFP1, 1999, pp. 1321-1326.

[25] S. Yoshioka, S. Obi, S. Masuda, Organized vortex motion in periodically perturbed turbulent separated flow over a backward-facing step, Int. J. Heat and Fluid Flow (2001) 301-307.

[26] S. Yoshioka, S. Obi, S. Masuda, Turbulence statistics of periodically perturbed separated flow over backward-facing step, Int. J. Heat and Fluid Flow (2001) 393-401. 
[27] A. Dejoan, M. Leschziner, Large eddy simulation of periodically perturbed separated flow over a backward-facing step, Int. J. Heat and Fluid Flow (2004) 581-592.

[28] Z. Mehrez, M. Bouterra, A. E. Cafsi, A. Belghith, P. L. Quéré, Simulation of the periodically perturbed separated and reattaching flow over a backward-facing step, Journal of Applied Fluid Mechanics 3 (2010) $1-8$.

[29] S. Obi, Case 9.3: Periodically perturbed separated flow over backward-facing step, in: Contributed papers of the 5th ERCOFTAC/IAHR Workshop on Refined Tutrbulence Modeling, October 4-5, 2001, Darmstadt, Germany.

[30] G. H. Rhee, H. J. Sung, Numerical prediction of locally forced turbulent separated and reattaching flow, Fluid Dynamics Research 26 (2000) 421-436.

[31] S. Saric, S. Jakirlic, C. Tropea, A periodically perturbed backward-facing step flow by means of LES, DES and T-RANS: An example of flow separation control, J. of Fluids Engineering (2005) 879-887.

[32] E. Creusé, A. Giovanni, I. Mortazavi, Vortex simulations of active control strategies for transitional backward-facing step flows, Comput. Fluids 38 (2009) 1348-1360.

[33] A. Martin-Alcántara, E. Sanmiguel-Rojas, Gutiérrez-Montes, Martinez-Bazán, Drag reduction induced by the addition of a multi-cavity at the base of a bluff body, J. of Fluids and Structures 48 (2014) $347-361$.

[34] M. Rouméas, P. Gilliéron, A. Kourta, Analysis and control of the near-wake flow over a square-back geometry, Comput. Fluids 38 (2009) 60-70.

[35] S. Ahmed, G. Ramm, G. Faltin, Salient features of the time-averaged ground vehicle wake, SAE Technical Paper 840300, 1984.

[36] B. Basara, Case 9.4 : Flow around a simplified car body (Ahmed body), in: 10th joint ERCOFTAC (SIG15) -IAHR-QNET/CFD Workshop on Refined Turbulence Modelling, October 10-11, 2002, University of Poitiers, France.

[37] E. Serre, M. Minguez, R. Pasquetti, E. Guilmineau, G. Deng, M. Kornhaas, M. Schaefer, J. Froehlich, C. Hinterberger, W. Rodi, On simulating the turbulent flow around the Ahmed body: a French-German collaborative evaluation of LES and DES, Comp. \& Fluids 78 (2013) 10-23. 
[38] M. Grandemange, M. Gohlke, O. Cadot, Turbulent wake past a three-dimensional blunt body. part 1. global modes and bi-stability, J. Fluid Mech. 722 (2013) 51-84.

[39] J. Östh, B. Noack, S. Krajnovic, D. Barros, J. Borée, On the need for a nonlinear subscale turbulence term in POD models as exemplified for a high-Reynolds-number flow over an Ahmed body, J. Fluid Mech. 747 (2014) 518-544.

[40] R. Volpe, P. Devinant, A. Kourta, Private communication, ANR project LIVE-CAMS, 2013.

[41] C. Chok, S. Parameswaran, R. Sun, M. Gleason, Numerical investigation of the effects of base slant on the wake pattern and drag of three-dimensional bluff bodies with a rear blunt end, J. Wind Eng. Ind. Aerodyn. 21 (1994) 417-426.

[42] Y. Eulalie, Etude aérodynamique et contrôle de la traînée sur un corps de ahmed culot droit, Ph.D. thesis, Bordeaux University (2014). 